\title{
Rumen Sampling Methods Bias Bacterial Communities Observed
}

2

3 Jill V. Hagey ${ }^{1}$,

4 Maia Laabs ${ }^{1}$,

5 Elizabeth A. Maga ${ }^{1}$

6 Edward J. DePeters ${ }^{1}{ }^{*}$

7

$8 \quad{ }^{1}$ Department of Animal Science, University of California Davis, California, USA

9

10 Running title: Biases of Rumen Sampling Methods

11

$12 *$ Correspondence:

13 Edward J. DePeters

14 ejdepeters@ucdavis.edu

15

16

17

18

19

20

21

22

23

24

25

26

27

28

29 


\section{Abstract}

31 The rumen is a complex ecosystem that plays a critical role in our efforts to improve feed

32 efficiency of cattle and reduce their environmental impacts. Sequencing of the 16S rRNA gene

33 provides a powerful tool to survey shifts in the microbial community in response to feed

34 additives and dietary changes. Oral stomach tubing a cow for a rumen sample is a rapid, cost-

35 effective alternative to rumen cannulation for acquiring rumen samples. In this study, we determined how sampling method, as well as type of sample collected (liquid vs solid), bias the microbial populations observed. The abundance of major archaeal populations was not different at the family level in samples acquired via rumen cannula or stomach tube. Liquid samples were enriched for the order WCHB1-41 (phylum Kiritimatiellaeota) as well as the family Prevotellaceae and had significantly lower abundance of Lachnospiraceae compared with grab samples from the rumen cannula. Solid samples most closely resembled the grab samples; therefore, inclusion of particulate matter is important for an accurate representation of the rumen microbes. Stomach tube samples were the most variable and were most representative of the liquid phase. In comparison with a grab sample, stomach tube samples had significantly lower abundance of Lachnospiraceae, Fibrobacter and Treponema. Fecal samples did not reflect the community composition of the rumen, as fecal samples had significantly higher relative abundance of Ruminococcaceae and significantly lower relative abundance of Lachnospiraceae compared with samples from the rumen.

\section{Introduction}

The ruminant stomach consists of four chambers the reticulum, rumen, omasum, and abomasum. The rumen, which is the largest of the four compartments, is a complex pregastric 
52 anaerobic fermentation chamber that harbors a diverse microbial community of bacteria,

53 archaea, protozoa, and fungi [1]. These microbes exist symbiotically inside the ruminant host and

54 are responsible for fermentation of dietary compounds. During the anaerobic fermentation of

55 chemical constituents in the diet, volatile fatty acids (VFA), B-vitamins, and microbial cell

56 proteins are produced, which serve as sources of nutrients and energy for the host that have a

57 direct effect on physiological and production parameters [2]. As the rumen compartment does

58 not secrete enzymes, ruminants are dependent on the enzymes produced by the various rumen

59 microbes for digestion of feed. These microbial enzymes allow the ruminant to convert a wide

60 variety of both plant- and animal-based feedstuffs into products that will contribute to the

61 synthesis of meat and milk for human consumption. The bacterial population of the rumen

62 comprises nearly $95 \%$ of the total microbial community and is diverse. There are many genera of

63 bacteria that have been linked to feed efficiency, milk yield, and milk composition in dairy cattle

$64[3,4]$.

Factors such as age [5], breed [6-8], health status, season [9] and diet of the animal all contribute to variation in the microbiota of the rumen. Dietary composition was reported to be

67 the primary factor affecting the taxa present in the rumen microbiota as well as the richness of those taxa, with the ratio of forage-to-concentrate in the diet of utmost importance [10-12]. The rumen is home to a stable yet dynamic microbial ecosystem that has adapted to survive in an anaerobic environment with osmotic pressure, high buffering capacity and internal competition

71 for substrate [13]. When dietary changes occur slowly, rumen conditions change causing

72 microbial populations to shift in response to the new feed ingredients by favoring the growth of

73 certain taxa over others, which subsequently affects the organic acid profiles produced [14].

74 However, when a dietary change occurs rapidly, for example changing from high forage diet 
75 (high cellulose and hemicelullose substrate) to a high concentrate diet (high starch and sugar),

76 the shift in microbial community often causes simple indigestion in the cow, which can lead to

77 ketosis. This occurs in dairy production when cows transition from a high forage diet fed

78 prepartum to a lower forage, higher concentrate lactation diet in a matter of hours, which can

79 contribute to indigestion. Management strategies at the farm level have evolved to minimize

80 perturbations to the rumen microbial environment that reduce health and production performance

81 when ingredients in the diet change due to cost or availability of a feed ingredient. Methods to

82 quickly sample and diagnose microbial perturbations due to dietary transitions could improve the management strategies of these high-risk animals.

The composition of the rumen microbiota was first described by Hungate in 1966, and

85 has been studied more extensively in recent years, in part due to the reduced costs associated

86 with next generation sequencing techniques such as the pyrosequencing and Illumina platforms

87 [15]. Much of the recent interest in the rumen microbiota has been generated by research related to climate change and the potential to reduce methane emissions from ruminant livestock as a greenhouse gas mitigation strategy. Next generation sequencing has thus far been a successful tool for characterizing the diversity of the microbial community within the rumen in greater

91 detail through 16S rRNA gene amplicon profiling [16,17]. This technology is advantageous in

92 that it allows the identification of a broader array of rumen microbial taxa, given that only a

93 small fraction of the total species have been successfully cultured. However, the most

94 appropriate method of obtaining a representative rumen sample is still widely debated [18]. It is

95 well known that the bacterial populations between the solid and liquid portions of the rumen

96 digesta differ in microbial composition, suggesting that the sampling method used will affect the 
97 characterization of the microbial community [19-24]. Thus, identifying sampling methods that

98 accurately represent both the liquid and solid fractions of the rumen digesta are necessary. Much of the existing research describing the rumen microbiome was performed on animals surgically fitted with rumen cannula, which offer the accuracy and convenience of sampling both liquid and solid rumen digesta directly from the rumen chamber. However, the surgical fistulation procedure is invasive, and the costs associated with the procedure as well as the ongoing animal care limit the number of animals that can feasibly be used in an experiment. Importantly, if microbial biomarkers of health or disease are identified for on-farm testing, retrieving rumen fluid through a cannula is not a practical approach on commercial dairy and livestock farms. Alternatively, many studies have used an oral stomach tube to collect rumen fluid without the need for a rumen fistula $[8,18,25]$. Oral stomach tubes are a cheaper, less invasive approach to rumen sampling that can be performed on as many cows as necessary, thus economically increasing the experimental sample size. In terms of bacterial community composition and diversity, rumen fluid extracted via the fistula was comparable to fluid extracted via the oral stomach tube $[8,26]$. Some of the disadvantages of the oral stomach tube include possible contamination by saliva (which affects the $\mathrm{pH}$ of the sample), inconsistent sampling region within the rumen, stress to the animal, skilled labor associated with use, and limited representation of particulate matter in samples, though the importance of these concerns to the microbial composition of the sample are widely debated among researchers [18,27].

The collection of fecal material from cattle is another non-invasive, simple, and inexpensive technique that is not as commonly regarded as a viable tool for collecting samples representative of the rumen microbiota. Although fecal sampling requires minimal equipment, is cost-effective, and can be performed easily on any animal, bacterial populations of the feces 
were found to not reflect the rumen digesta $[28,29]$. However, in these studies, the fecal microbiome was not compared with the liquid and solid fractions of the rumen digesta individually. If the feces reflect the microbial populations in the solid fraction, fecal samples might be useful in evaluating microbial taxa involved in fiber digestion. Conversely, if fecal samples represent the liquid fraction, lactate-producing microbes that contribute to ruminal acidosis could be diagnosed in a less invasive manner.

The aim of this study was to identify and compare the bacterial populations present in samples collected using three methods - an oral stomach tube, fecal samples, and grab sample through a rumen fistula. To the authors' knowledge, no studies have considered this variety of sampling methods on a comparative basis using next generation sequencing. Our results will be useful in helping investigators design experiments that capture their microbial populations of interest.

\section{Materials and methods}

\section{Animals}

The experimental protocol and all procedures used in this study were approved by the UC Davis Institutional Animal Care and Use Committee. Four non-lactating Holstein (3) and Jersey (1) cows, each ruminally fistulated prior to the study, were used for the collection of samples. For the two-week duration of the study, cattle were housed individually with ad libitum access to water and offered the same maintenance total mixed ration (TMR) twice daily at approximately 08:00 and 16:00. Dietary composition of TMR was analyzed for protein, fiber, mineral, and energy content (Cumberland Valley Analytical Services, Hagerstown, MD; Table 1).

\section{TABLE 1 | Dietary Composition of Total Mixed Ration}

\begin{tabular}{|l|l} 
Item $^{\text {a }}$ & Dietary Composition $^{\text {b }}$ \\
\hline
\end{tabular}




\begin{tabular}{|c|c|c|}
\hline 141 & Moisture & $11.1 \%$ \\
\hline 142 & Dry matter & $88.9 \%$ \\
\hline 143 & Crude protein & $10.7 \% \mathrm{DM}$ \\
\hline 144 & Adjusted protein & $91.4 \% \mathrm{CP}, 9.8 \% \mathrm{DM}$ \\
\hline 145 & Soluble protein & $28.6 \% \mathrm{CP}, 3.1 \% \mathrm{DM}$ \\
\hline 146 & ADF & 78.5\% NDF, 36.7\% DM \\
\hline 147 & aNDF & $46.8 \% \mathrm{DM}$ \\
\hline 148 & Ash & $9.62 \% \mathrm{DM}$ \\
\hline 149 & Calcium & $0.33 \% \mathrm{DM}$ \\
\hline 150 & Phosphorus & $0.22 \% \mathrm{DM}$ \\
\hline 151 & Magnesium & $0.25 \% \mathrm{DM}$ \\
\hline 152 & Potassium & $1.91 \% \mathrm{DM}$ \\
\hline 153 & Sodium & $0.08 \% \mathrm{DM}$ \\
\hline 154 & Iron & 449 ppm \\
\hline 155 & Zinc & $54 \mathrm{ppm}$ \\
\hline 156 & Copper & $8 \mathrm{ppm}$ \\
\hline 157 & TDN & $57.5 \% \mathrm{DM}$ \\
\hline 158 & Net energy lactation & $0.59 \mathrm{Mcal} / \mathrm{lb}$ \\
\hline 159 & Net energy maintenance & $0.56 \mathrm{Mcal} / \mathrm{lb}$ \\
\hline 160 & Net energy gain & $0.30 \mathrm{Mcal} / \mathrm{lb}$ \\
\hline 161 & Non fiber carbohydrates & $32.9 \% \mathrm{DM}$ \\
\hline
\end{tabular}

164 Table 1: Chemical composition of the total mixed-ration (TMR) fed to the rumen-fistualted dry 165 cows. Dietary analysis conducted by Cumberland Valley Analytical Services (Hagerstown, MD) 166 completed 12/01/2016. Ingredient composition of TMR on an as is a basis was 50\% wheat hay, $16725 \%$ alfalfa hay, $21.4 \%$ almond hulls and 3.6\% mineral supplement. ${ }^{a}$ Acid detergent fiber 
(ADF); Ash free Neutral Detergent Fiber (aNDF); Total Digestible Nutrients (TDN). ${ }^{b}$ Dry

Matter (DM); Crude Protein (CP); Neutral Detergent Fiber (NDF).

170

171

172

173

174

175

176

177

178

179

180

181

182

183

184

185

186

187

188

189

190

\section{Sampling}

Cows were given a one-week period for environmental adaption prior to sampling. This

adaptation period was necessary to allow them to acclimate to an individual (rather than group)

feeding approach and to reduce sorting of the feed. All cows were fed the maintenance TMR diet

(Table 1) prior to and throughout the study. Sampling of fecal and ruminal contents occurred on

days 7, 9, and 11 of the experiment, and took place approximately 4 hours after morning feeding.

Fecal samples were collected from the rectum with sterile polyethylene gloves and stored in

plastic bags. Grab samples (containing both liquid and particulate matter) from the fistula were

collected from the medioventral region of the rumen and stored in plastic bags. Rumen liquid

was collected from the fistula using a PVC pipe, Tygon ${ }^{\circledR}$ tubing, and a large syringe, and stored

in $240 \mathrm{ml}$ sterile plastic vials. The Tygon ${ }^{\circledR}$ tubing was thoroughly rinsed and bleached between

cows to avoid cross-contamination of samples. For liquid strained samples, about $250 \mathrm{ml}$ of

liquid sample was squeezed through 4 layers of cheesecloth to remove large particles, as is

common done [19,30,31]. For solid samples, similar squeezing through a cheese cloth was

applied to remove liquid from the solid digesta content, before being stored in plastic bags. On

days 9 and 11, the first aliquot of rumen liquid, containing both liquid and solid particulates, was

additionally collected as a liquid unstrained sample in $240 \mathrm{ml}$ sterile plastic vials. Lastly, enough

rumen liquid to fill a $240 \mathrm{~mL}$ sterile plastic vials, which was collected via an oral stomach tube

using an oral speculum, Tygon ${ }^{\circledR}$ tubing (1.5cm O.D. and $0.9 \mathrm{~cm}$ I.D.) and a vacuum pump. A

fresh tube was used for each cow to avoid cross-contamination of samples. The $\mathrm{pH}$ of each of the liquid-containing samples was measured with a portable $\mathrm{pH}$ meter (Milwaukee Instruments, 
191 Rocky Mount, NC). All samples were held on ice during transport and stored in triplicate $60 \mathrm{ml}$

192 vials at $-20^{\circ} \mathrm{C}$ for DNA extraction and dry matter analysis.

193

195

196

197

198

199

200

201

202

203

204

205

206

207

208

209

210 211 via the Illumina MiSeq PE250 platform (Illumina, CA).

\section{Amplicon library processing}

DNA extraction was performed using a ZR Fecal DNA MiniPrep ${ }^{\mathrm{TM}}$ kit (Zymo Research Corp., Irvine, CA), with slight modifications to the manufacturer's instructions. Samples were thawed at room temperature, and $200 \mathrm{mg}$ of each sample were used for DNA extraction, which included a bead bashing step to facilitate the mechanical lysis of microbial cell walls. As the last step in the procedure, DNA was eluted from the column with elution buffer, and the resulting DNA was evaluated for concentration and purity on a NanoDrop 2000 spectrophotometer (Thermo Scientific, Waltham, MA, USA) and stored at $-20^{\circ} \mathrm{C}$. The V4 region of the bacterial 16S rRNA gene was amplified from each sample using forward primer F515 containing a unique 8 bp barcode $(\mathrm{N})$ and linker region (GT) (5'-NNNNNNNNGTGTGCCAGCMGCCGCGGTAA3') and the reverse primer R806 (5'-GGACTACHVGGGTWTCTAAT-3'). The amplification was carried out in triplicate using GoTaq ${ }^{\circledR}$ Green Master Mix (Promega, Madison, WI) as previously described [32]. In brief, PCR conditions were set at initial denaturation for $94^{\circ} \mathrm{C}$ for 3 min; followed by 35 cycles of $94^{\circ} \mathrm{C}$ for 45 seconds, $50^{\circ} \mathrm{C}$ for $1 \mathrm{~min}, 72^{\circ} \mathrm{C}$ for 90 seconds with final extension step at $72^{\circ} \mathrm{C}$ for $10 \mathrm{~min}$. Triplicates were combined in equal concentrations and amplicons were evaluated for off target bands by gel electrophoresis, pooled and then purified using a QIAquick PCR Purification Kit (QIAGEN, Hilden, Germany). A 50 $\mu 1$ aliquot of the final pooled PCR product was sequenced at the UC Davis Genome Center DNA Technologies Core 
Raw paired end reads were screened to remove phiX, human and host contamination

214

215

216

217

218

219

220

221

222

223

224

225

226

227

228

229

230

231

232

233

234

235

using Kneaddata v0.6.1 by aligning reads to the phiX174 (NCBI ACC: NC_001422.1), bovine (ARS-UCD1.2) and human (GRCh38) reference genomes [33]. Reads were demultiplexed followed by trimming of primers and barcodes with Cutadapt v1.18 [34]. Ends of reads were trimmed for quality, any read smaller than $150 \mathrm{bp}$ was discarded and a max expected error of 2 was used as a quality filter using the filterAndTrim function from DADA2 v1.8.0 [35].

Sequences were merged, denoised, chimeras were removed and exact amplicon sequence variants (ASVs) were identified using DADA2. Taxonomy was assigned using the RDP native Bayesian classifier algorithm in the DADA2 assignTaxonomy function with Silva reference database v.132 training set. A phylogenetic tree of unique ASVs was made using FastTree with default options in QIIME v.1.9.1 [36]. The ASV table, sequences and tree produced by DADA2 were imported into the R package Phyloseq v.1.24.2 for further analysis [37].

\section{Microbial community analyses and statistics}

First, unsupervised exploratory analysis was conducted with double principal coordinates analysis (DPCoA), which was calculated and graphed with the phyloseq R package $[37,38]$. Both modeling and hypothesis testing of differentially abundant ASVs between sample types was determined using the Corncob R package [39]. All genera-level and ASV-level relative abundances were modeled using a beta-binomial regression with a logit-link for mean and dispersion as described by Martin et al [39]. Differential abundance was modeled as a linear function of sample type, cow and day. Significant differentially abundant ASVs were determined with the parametric Wald test with bootstrapping ( $n=1000)$ as described by Martin et al [39].

Within the Corncob algorithm the Benjamini-Hochberg $(\mathrm{BH})$ adjustment for multiple comparisons was used to calculate adjusted $p$ values. An adjusted $p$ value $\leq 0.05$ was considered 
236 significant. This model has the benefit of accommodating the absence of a taxon in samples

237 without zero-inflation or pseudocounts, accounts for differences in library sizes, give valid

238 inference even with small samples [39]. Richness of sample types was estimated with the R

239 package breakaway and evenness was calculated using the R package DivNet, which accounts

240 for the structure of microbial communities [40,41]. Hypothesis testing of alpha diversity

241 (richness and evenness) metrics was done using the betta() function using sample type, cow and

242 day as fixed effects in the breakaway R package [42]. Beta diversity was calculated by using

243 unweighted UniFrac distances and graphed by PCA clustering in the Phyloseq R package [37].

244 The number of clusters in the data was determined with the gap statistic using the gapstat_ord()

245 function in Phyloseq [43].

246 Data availability

247 Scripts for sequence processing and analysis, interactive graphs, R objects as well as an

248 Rmarkdown file to reproduce figures in this paper can be found

249 at https://doi.org/10.5281/zenodo.4026849. Raw sequencing files are available through the

250 Sequence Read Archive under the study accession number PRJNA692782.

251 Results

252 Sequence processing of rumen and fecal samples

253 After filtering with Kneaddata and demultiplexing the single run of MiSeq yielded

$254747,961250 \mathrm{bp}$ raw paired-end reads that entered the DADA2 pipeline. After the quality

255 trimming, initial filtering, and chimera removal, the library size ranged from 2,189 to 24,624

256 reads, with a median library size of 7,197 and an average size of 8,110 reads. The median read

257 length of quality filtered merged reads was 257bp. A total of 5,607 AVSs were identified, of 
which 94 weren't assigned to a phylum and thus were removed for analysis along with 12 ASVs

259

260

261

262

263 assigned to chloroplasts and mitochondria. The 94 unassigned taxa were found in all sample types with solid samples having the most reads of unknown taxa. This suggests there are is still a diverse group of microbes attached to solid particles that have yet to be identified. The final feature table had 5,485 ASVs across 68 samples.

\section{Community composition of all sample types}

The 5,485 ASVs were assigned to 21 phyla, 78 orders, 117 families, and 293 genera.

Here we define major phyla as those with a mean relative abundance in at least one sample type of greater than 3\%. Major phyla were Firmicutes, Bacteroidetes, Kiritimatiellaeota, Proteobacteria, Euryarchaeota and Spirochaetes (Fig 1A). Of these major phyla, Firmicutes was significantly lower in relative abundance $(P \leq 0.0001$; Fig $1 \mathrm{C})$ while Bacteroidetes and Proteobacteria had significantly higher relative abundance in feces and liquid samples as compared with grab samples $(P \leq 0.002$; Fig $1 \mathrm{C})$. In addition, Kiritimatiellaeota was significantly higher in relative abundance in stomach tube and liquid samples compared to grab samples $(P \leq 0.001$; Fig $1 \mathrm{C})$. Spirochaetes was significantly lower in relative abundance in feces, stomach tube and solid samples compare with grab samples $(P \leq 0.003$; Fig $1 \mathrm{C})$. While Euryarchaeota had significantly lower relative abundance in feces, it had significantly higher relative abundance in stomach tube samples compared with grab samples $\left(P=3.24 \times 10^{-8}\right)$. Minor phyla were those with less than 3\% relative abundance in all samples (Fig 1B). An interactive version of Figure 1B with mean and standard deviations for each phyla is available at https://doi.org/10.5281/zenodo.4026849 as interactive Fig 1 - minor phyla. The phylum Gemmatimonadetes was only found in stomach tube samples and Deferribacteres was only found in fecal samples (Figure 1B). For the minor phyla in feces Tenericutes, Patescibacteria, 
Actinobacteria, Fibrobacteres, Chloroflexi and Synergistetes were significantly lower in relative abundance and Verrucomicrobia, Epsilonbacteraeota, Cyanobacteria, Planctomycetes and Lentisphaerae were significantly higher in relative abundance compared with grab samples $(P \leq$ 0.001; Figure 1C). Samples acquired with the oral stomach tube had significantly lower relative abundance of Patescibacteria and Fibrobacteres and significantly higher relative abundance of Verrucomicrobia, Epsilonbacteraeota, and Fusobacteria compared with grab samples. Only $1.68 \%$ of ASVs were assigned a species, but $67.3 \%$ were able to be assigned to a genus.

Fig 1. Relative abundance of (A) major and (B) minor phyla and (C) their differential

abundances. (A) Relative abundance of major phyla defined as those phyla found at greater than grab samples. type.

\section{Diversity}


The evenness of fecal samples was significantly lower than all rumen sample types $(P \leq$

305

306

307

308

309

310

0.001; Fig 2A). Fecal, stomach tube, and liquid strained samples had significantly lower

evenness than grab samples $(P \leq 0.001 ;$ Fig $2 \mathrm{~A})$. Solid and liquid unstrained samples did not

have significantly different evenness compared with grab samples ( $P \geq 0.05$; Fig $2 \mathrm{~A})$. Both the individual cow sampled and day of sampling significantly affected the evenness of a sample $(P \leq$ 0.05; Fig 2A).

Fig 2. Differences in estimated alpha diversity among sample types. (A) DivNet estimate of Shannon diversity plotted as mean with a $95 \%$ confidence intervals and (B) mean breakaway estimate of species richness with $95 \%$ confidence intervals. Both the richness and evenness of fecal samples were significantly lower than all other rumen sample types $(\mathrm{P} \leq 0.001)$. Stomach tube and Liquid strained samples had significantly lower evenness than grab samples $(\mathrm{P} \leq$ 0.001). Solid and stomach tube samples were estimated to have significantly fewer species than grab samples ( $\mathrm{P}=0.02$ and $\mathrm{P} \leq 0.001$, respectively).

The richness of samples from the rumen were estimated to be significantly higher than that of fecal samples $(P \leq 0.001$; Fig 2B). Fecal samples were estimated to have a mean of 2,021 species, which was significantly lower than the grab samples estimated mean of 4,119 species $(P$ $\leq 0.001$; Fig 2B). Liquid strained and unstrained samples did not have a significantly different mean number of estimated species compared with grab samples ( $P \geq 0.05$; Fig $2 \mathrm{~B})$. However, solid and stomach tube samples were estimated to contain a significantly lower number of species compared with grab samples, 286 and 506, respectively $(P=0.02, P \leq 0.001$; Fig 2B). Neither the day sampled nor individual cow had a significant effect on the number of species in a sample $(P \geq 0.05$; Fig 2B). 
Weighted UniFrac distances were calculated to determine beta diversity. Calculations of

327

328

329

330

331

332 eigenvalues showed that $86.8 \%$ of the variance between samples was contained in the first two principle components, thus a two-dimensional visualization was deemed appropriate (Fig 3).

Two distinct groups were present with fecal samples clustering away from all rumen sample types (Fig 3). Grab and solid samples exhibited low variability and overlapped each other, forming one group. Liquid samples were further down the second axis, which might indicate that there were distinct phylogenetic differences between these samples and grab samples. Stomach tube samples were the most variable with some of these samples found within the grab and solid sample cluster, while other stomach tube samples were more closely associated with liquid samples. The gap statistic of the weighted UniFrac strongly suggested there were at least 3-5 clusters in the data. As there are six sample types in the dataset, this suggests that grab and solid samples are likely one cluster as these samples overlap the most (Fig 3). The unweighted UniFrac showed a similar pattern, with less variation explained in axis one and two, $45.8 \%$ and $6.9 \%$, respectively (data not shown).

Fig 3. Beta diversity as weighted UniFrac distances between samples. To faithfully reflect the variance in the coordinates, the height-to-width ratio was based on the ratio between the corresponding eigenvalues.

\section{Overall differences between sample types}

As an exploratory first step, DPCoA was performed (Fig 4A). An interactive version of this graph with taxon identification is available at https://doi.org/10.5281/zenodo.4026849 as interactive figure 2 - DPCoA. Additionally, since Firmicutes and Bacteroidetes dominated a majority of the graph, a version without these phyla was created at and is available at https://doi.org/10.5281/zenodo.4026849 as interactive Fig 3 - DPCoA_NoFrimBact with the aim 
to allow a better visualization of minor phyla. This phylogenetic ordination method provides a biplot representation of both samples and taxonomic categories. The DPCoA was used to identify the underlying structure of these data and identify taxa that could be contributing to differences between sample types that will be specifically examined with differential abundance testing.

\section{Fig 4. Double principal coordiant analysis (DPCoA) of the Bray-Curtis distances among}

1. The $1^{\text {st }}$ axis discrimates fecal from rumen samples while the $2^{\text {nd }}$ axis separtes liquid strained samples from other rumen sample types. Samples that have larger scores on CS1 have a subset of taxa from Bacteroidetes and Firmicutes that is different than rumen samples. Liquid strained samples have lower values on CS2 suggesting they are distinguished from other rumen sample types by taxa in the phylum Kiritimatiellaeota and Prevotellaceae. Fecal samples are predicted to have lower abundance of Lachnospiraceae and greater abundance of Ruminococcaceae.

367 the phylum Bacteroidetes, mainly Rikenellaceae and Prevotellaceae on the $1^{\text {st }}$ axis (Fig 4B).

368 Additionally, fecal samples separate from samples from the rumen based on having more taxa

369 from the family Akkermansiaceae and phylum Tenericutes and fewer from the families

371 on the $2^{\text {nd }}$ axis of the DPCoA, indicating these samples had more taxa from the phylum 
372 Kiritimatiellaeota and a subset of Bacteroidetes most of them in the family Prevotellaceae (Fig

373 4B). Also, the separation of liquid strained samples away from other rumen samples was due to

374 fewer taxa from the phylum Euryarchaeota and the family Eggerthellaceae that is within the

375 phylum Actinobacteria (S1 Fig).

To test the significance of these differences, differential abundance testing was performed

377 with Corncob. All sample types were compared to grab samples as a base line, because it is

378 considered the gold standard for surveying microbial communities in the rumen. The relative

379 abundance of Prevotellaceae was significantly lower in feces and was significantly higher in

380 liquid samples compared with grab samples $(P \leq 0.0004$; Fig 5A and 6). Stomach tube $(P=0.06)$

381 and solid $(P=0.77)$ samples were not significantly different in the relative abundance of

382 Prevotellaceae compared with grab samples. The relative abundance of Prevotellaceae was

383 highest in liquid strained samples compared with other sample types (Fig 5A). In comparison to

384 grab samples, the relative abundance of Ruminococcaceae was significantly higher in feces $(P \leq$

$385 \quad 0.001$; Fig 5B) and solid $(P=0.003$; Fig 5B) samples while liquid strained samples had

386 significantly lower relative abundance $(P \leq 0.001$; Fig $5 \mathrm{~B})$. Neither stomach tube nor liquid

387 unstrained samples had significantly different relative abundance of Ruminococcaceae compared

388 with grab samples. Fecal samples were lower in relative abundance of Lachnospiraceae

389 compared with all other samples $\left(P \leq 6.96 \times 10^{-15}\right)$, while relative abundance was higher for grab

390 samples compared with all other sample types $(P \leq 0.03$; Fig $5 \mathrm{C})$. Neither day of sampling nor

391 individual animal significantly affected the relative abundance of Prevotellaceae $(P \geq 0.05)$. In

392 contrast, the relative abundance of Ruminococcaceae and Lachnospiraceae was significantly

393 affected by individual animal $(P \leq 0.03)$, but not day of sampling. 
Fig 5. Significant differences in the relative abundance of specific bacterial families.

395

396

397

398

399

400

401

402

403

404

405

406

407

408

409

410

411

412

413

414

415

416

Relative abundance of (A) Prevotellaceae (B) Ruminococcaceae and (C) Lachnospiraceae as

modeled by corncob. Points are the estimated relative abundance and bars are a $95 \%$ prediction interval for each cow on different days of sampling.

\section{Specific community differences between grab and fecal samples}

To further distinguish what taxa were contributing to the separation of fecal samples from rumen samples on the DPCoA, we identified taxa that were found in one sample type and not the other. Within the phyla Firmicutes and Bacteroidetes, families Barnesiellaceae,

Chitinophagaceae, p-2534-18B5_gut_group, GZKB124, and Hymenobacteraceae were found in fecal samples, but were not found in grab samples. Conversely, Leuconostocaceae,

Carnobacteriaceae, Aerococcaceae, Syntrophomoadaceae, Bacteroidetes_DB2-2, PeH15, M2PB4-65_termite_group, $C O B \_P 4-1 \_t e r m i t e \_g r o u p$, Spirosomaceae, and Porphyromonadaceae were found in grab samples, but were not found in fecal samples.

Next, we identified ASVs, genera and families that were differentially abundant between sample types. There were 657 significant differentially abundant ASVs in fecal samples compared with grab samples, as well as 114 differentially abundant genera $(P \leq 0.05$; S2 Fig). At the genera level, 131 ASVs were unable to be fit to the Corncob model for differential abundance testing. Primarily, this was due to either limited or lack of reads in one of the sample types. Of these genera that didn't fit the model, Acetatifactor, Shuttleworthia, Succinivibrio, Veillonellaceae UCG-001, and Lachnospiraceae UCG-006 were found in all grab samples with greater than or equal to 50 reads across all samples, but absent in fecal samples. Similarly, there were 11 genera found in all fecal samples with 50 or more reads, but these were not found in any grab samples including Coprococcus 3, Cellulosilytium, Clostridioides, Paeniclostridium, 
417 Parasutterella, Aeriscardovia, Odoribacter, Harryflintia, Negativibacillus, Pygmaiobacter, and 418 Ruminococcaceae UCG-011.

The most common families with differentially abundant ASVs were Lachnospiraceae, Ruminococcaceae, Christensenellaceae, Family XIII, Rikenellaceae, and Prevotellaceae. These

421 families are in the phyla Firmicutes and Bacteroidetes, which had the most significant

422 differentially abundant ASVs. However, as a percent of total ASVs these phyla only had 4.9\%

423 and $16.3 \%$ significant differentially abundant ASVs, respectively. In contrast, $25.6 \%$ of the

424 ASVs assigned to Chloroflexi and 29.5\% of ASVs assigned to Euryarchaeota were significantly

425 different between grab and fecal samples. The significant ASVs in Chloroflexi were all assigned

426 to the genus Flexilinea. In addition to the significantly lower abundance of some Chloroflexi

427 ASVs in fecal samples compared with grab samples, another $51.3 \%$ of the ASVs in the phyla

428 were not found in any fecal samples (S2 Fig). In the phylum Euryarchaeota, feces had

429 significantly lower abundance of Methanobrevibacter, Methanosphaera, and were almost devoid of Methanomethylophilaceae.

There were 30 families that had significantly lower relative abundance while 18 families strongest positive relationship with fecal samples were Peptostreptococcaceae $\left(P=1.76 \times 10^{-7}\right.$;

434 Fig 7A), Akkermansiaceae $\left(P=6.95 \times 10^{-5}\right.$; Fig 7B $)$, and Bacteroidaceae $\left(P=7.87 \times 10^{-12}\right.$; Fig

435 7C), which were significantly higher in relative abundance compared with grab samples.

436 Conversely, the families with largest negative relationship between fecal and grab samples that

437 had significantly lower relative abundance were Veillonellaceae $\left(P=1.66 \times 10^{-11}\right.$; Fig 7D) and

438 Bacteroidales_BS11_gut_group $\left(P=6.20 \times 10^{-11}\right.$; Fig 7E). Additionally, fecal samples separated

439 from rumen samples on the DPCoA (S1 Fig) due in part to differences in the families 
Spirochaetaceae and Fibrobacteraceae both of which had significantly lower relative abundance than grab samples $\left(P=7.88 \times 10^{-9} ; P=4.73 \times 10^{-8}\right.$, respectively Fig $\left.7 \mathrm{~F}\right)$.

Fig 6. Families that were significantly differentially abundant across sample type compared with grab samples. Graphed as coefficients with a $95 \%$ confidence interval calculated from the corncob model. Families with negative coefficients for a sample type are expected to have a lower relative abundance when compared to the grab samples while positive coefficients suggest a higher relative abundance in that sample type compared to grab samples.

\section{Fig 7. Significant differences in the relative abundance of specific bacterial families between}

fecal and grab samples. Fecal samples had significantly higher relative abundance of (A)

Peptostreptococcaceae, (B) Akkermansiaceae, (C) Bacteroidaceae, compared to grab samples.

Also, there was significantly lower relative abundance of (D) Veillonellaceae, (E)

Bacteroidales_BS11_gut_group and (F) Spirochaetaceae compared to grab samples. Points are the estimated relative abundance and bars are a 95\% prediction interval for each cow on different days of sampling.

Based on the DPCoA findings, the phyla Spirochaetes and Actinobacteria also played an important role in distinguishing feces from grab samples (Fig 1A and 4B). In the phylum

Spirochaetes, there were 10 ASVs, all of which were from the genera Treponema_2, that had significantly lower relative abundance in fecal samples compared with grab samples. Within the phylum Actinobacteria, there were 4 ASVs in the genera Olsenella, 5 ASVs in Atopobium, 7 ASVs in the genera DNF00809, and 1 ASV assigned to Raoultibacter, which were all significantly lower in relative abundance compared with grab samples. 


\section{Specific community differences between grab and stomach tube}

463

464

465

466

467

468

469

470

471

472

473

474

475

476

477

478

479

480

481

482

483

484

\section{samples}

Oral stomach tube samples were composed of 20 phyla, 65 orders, 98 families, and 236 genera. There were $255 \mathrm{ASV}$ found in grab samples that were not found in the stomach tube samples. Likewise, 404 ASVs in stomach tube samples were not present in the grab samples.

There were 3,615 ASVs that were in common between stomach tube and grab samples. Three families Rhodobacteraceae, Bacteriovoracaeae, and Spirosomaceae were found in grab samples, but were not present in stomach tube samples. The 5 families found in stomach tube samples, but not in grab samples were Cellvibrionaceae, Neisseriaceae, Bifidobacteriaceae, Micrococcaceae, and Solirubrobaceraceae.

In addition to the taxa not found in a particular sample type, there were 13 families, 43 genera, and 199 ASVs significant differentially abundant between stomach tube and grab samples. Lachnospiraceae, Ruminococcaceae, Prevotellaceae, and Erysipelotrichaceae were the most common families to have significant differentially abundant ASVs in stomach tube versus grab samples. The relative abundance of 39 ASVs in the family Lachnospiraceae were significantly lower while 15 were significantly higher in comparison to grab samples. At the genus level, 15 genera in the family Lachnospiraceae were significantly lower in abundance, while Blautia, Acetitomaculum and Howardella were the only genera that had higher relative abundance (S2 Fig). While Ruminococcaceae in stomach tube samples was not significantly different from grab samples at the family level (Fig 5B), eight genera in this family were significantly higher in relative abundance between the two sample types. Prevotellaceae in stomach tube samples was not significantly different from grab samples at the family level $(P=$ 0.055; Fig 5A), but at the genus level, two were significantly lower and one significantly higher. 
Three genera in the family Erysipelotrichaceae, Catenisphaera, Erysipelotrichaceae _UCG-009, and Erysipelotrichaceae_UCG-004 were all significantly higher in stomach tube compared with grab samples. The only assigned genera in the family Fibrobacteraceae, Fibrobacter, was significantly lower in abundance in stomach tubes compared to grab samples (S1 Fig). The genus Streptococcus had significantly higher relative abundance compared with grab samples (Fig 6). The only genus in the phylum Euryarchaeota that had significant differences in abundance in samples from the stomach tube as compared with those collected from the rumen was Methanobrevibacter. This genus was significantly higher in stomach tube samples. At a finer resolution, there were only four ASVs assigned to Methanobrevibacter and one ASV assigned to Methnomethylophilaceae that were significantly higher in abundance in stomach tube samples compared with grab samples. However, at the family level three methanogenic families, Methanomethylophilaceae, Methanobacteriaceae and Methanocorpusculaceae, were not significantly different between the two sample types.

\section{Comparing sub-fractions of the grab sample}

Grab samples of rumen contents were placed in cheesecloth and squeezed to create the liquid strained sample and the solid particulate sample. There were 283 ASVs found in the grab sample that were not identified in the liquid strained samples. Conversely, there were 3,587 ASVs found in common between grab samples and liquid strained samples.

Based on the DPCoA, separation of liquid samples from other rumen sample types was driven in part by taxa from the phylum Kiritimatiellaeota (Fig 4). ASVs in this phylum were only assigned down to the order level with all ASVs assigned to WCHB1-41. Seventeen ASVs from Kiritimatiellaeota were significantly higher in liquid samples compared with grab samples while these ASVs were not significantly different in solid samples versus grab samples. 
518 abundance in Prevotellaceae than grab samples (Fig 5A). Within that family there was higher

525 were all within the order Gastranerophilales and were not classified any lower. Likewise, ASVs

527 significantly lower in abundance in liquid samples compared with grab samples (Fig 6). 
531 difference observed in the abundance of the genera Methanobrevibacter. Also, in the same

532 phylum Euryarchaeota, there was significantly lower relative abundance of Methanosphaera in

533 liquid strained samples when compared with grab samples.

\section{Discussion}

While other studies looked at differences in the rumen microbiome due to rumen

536 sampling method, they usually involved different diets and did not include all the sampling

537 methods presented in the current study. As diet is an important factor that affects the rumen

538 microbiome, we choose to keep the diet consistent during the study to fully investigate the

539 differences between sampling methods. To the authors' knowledge, this is the first study to

540 compare rumen sampling methods utilizing ASVs rather than OTUs. Therefore, this study has

541 the advantage of identifying AVS that are comparable across studies, which will improve the

542 reproducibility of sequencing studies of the rumen [44].

543 Kim et al. detected 19 bacterial phyla in the rumen with Firmicutes $(57.8 \%)$,

544 Bacteroidetes (26.7\%) and Proteobacteria (6.9\%) in greatest abundance with the remainder of the

54516 phyla less than $3 \%$ of the total sequences [45]. In the present study, 21 phyla were identified

546 in the grab sample and only three phyla were over 3\% abundance: Firmicutes (64.3\%),

547 Bacteroidetes (20\%) and Spirochaetes (4.1\%). This differed from fecal samples where the top

548 four phyla were Firmicutes (61.2\%), Bacteroidetes (32.1\%), Verrucomicrobia (1.3\%) and

549 Proteobacteria (1.1\%) with the remainder of the phyla observed at less than $1 \%$ mean relative

550 abundance (Fig 1A and 1B). The relative abundance of Firmicutes and Bacteroides in the fecal

551 samples were similar to what Wong et al. found in fresh manure, but they found Actinobacteria

552 among the top four phyla rather than Verrucomicrobia [46]. 
The day a sample was collected did not affect the number of species sampled and did not impact the abundance of Prevotellaceae, Ruminococcaceae and Lachnospiraceae. These observations agree with previous work that found there was little day-to-day variation in both the

556 solid and liquid fraction of rumen samples from the same animal [47]. Previous work found

557 differences between breeds [6,7], while others found minimal to no influence of breed $[8,48]$. As

558 we only had one Jersey as part of this study we are unable to determine the impact of breed on 559 the community composition.

\section{Diversity}

In the present study, fecal samples had lower richness when compared to grab samples.

562 This is in agreement with a study that used Faith's Phylogenetic Diversity to compare samples

563 from esophogeal tubing or feces of beef calves [49]. The same result was found using the

564 number of ASVs present in fecal compared to rumen condense after slaughter [50]. Similar to

565 fecal samples, we found that samples collected via the esophageal tube had lower richness than

566 grab samples. Such a finding was expected as microbes adhered to particles would be in low

567 proportion or excluded in the stomach tube sample, even though the tube used did not have a

568 screen. Using a stomach tube without a screen allowed the collection of small size particulates

569 only, whereas the grab samples included small to large particulate sizes. Our finding contradicts

570 Paz et al. who reported no difference in richness between a rumen sample collected from a

571 rumen cannula compared with a sample collected via esophageal tube [8]. However, in Paz et al.,

572 solid particles that adhered to the metal strainer of the esophageal tube were recovered and added

573 to the esophageal sample to create a sample that was "more adequately representative of the

574 rumen content", which suggests the authors acknowledge that a sample collected by an

575 esophageal tube that did not contain particles would not represent rumen contents. However, the 
research did not address this suggestion by analyzing the rumen contents collected with a stomach tube without the added solids.

Our work also differed from that of Ji et al. who reported the diversity of the bacterial population was not affected by sample type [51]. Samples in their study included rumen digesta collected from a cannula that was squeezed through cheese cloth to create a liquid and a solid fraction for comparison with rumen digesta from a cannula. However, we determined that both liquid sample types did not have significant differences in the number of taxa observed compared to grab samples, while solid samples had significantly lower estimated species than grab samples. The work of Weimer et al. (2017) used a sample cup to collect $100 \mathrm{ml}$ of digesta from the medio-ventral region of the rumen followed by squeezing through cheese cloth to create a liquid and a solid sample [31]. While this study found that community diversity and community richness were greater in solids than liquid, our data showed the opposite. Greater richness in liquid samples could potentially be explained by the greater relative abundance of Prevotellaceae, the most abundant species in the rumen, compared with the estimated number of species in solid samples. Jewel et al. found liquid samples to have higher richness than solid samples in agreement with our data [52].

Some of these discrepancies are in part due to differences in the metric used to estimate richness. All these previous studies reported Chaol as a measure of richness, but the current study used breakaway to estimate richness. Many alpha diversity estimates that are ubiquitous in the literature are highly biased and require statistical adjustments to address this bias, which Chaol does not [40,41]. Further, the strong negative bias of Chaol is even further increased by the use of rarefying as a means of normalization in the previous studies [53]. It is true that Dr. Anne Chao proposed that Chaol could be a useful metric for datasets that skewed toward low- 
abundance classes as microbiome data does; however, these low abundance counts aren't reliable due to sequencing platform and PCR errors. Breakaway addresses some short comings of Chao1 by providing an estimate of variance of richness estimates for hypothesis testing, estimating the number of missing taxa, and adjusting the richness estimate accordingly (bias correction) to provide a more accurate estimate of richness [41]. While this approach produces large error bars, the breakaway estimate provides a more accurate reflection of the uncertainly associated with estimating a true value that can never be known (Fig 2B).

\section{Bacterial populations}

\section{Rumen samples}

Based on the exploratory analysis with the DPCoA, differences between rumen liquid strained samples and other rumen samples types were driven mainly by Lachnospiraceae, Prevotellaceae and Kiritimatiellaeota. Lachnospiraceae was significantly lower in liquid samples and Prevotellaceae had significantly higher relative abundance compared with grab samples (Fig 5A and 5C). Other studies that examine differences between the microbial communities in liquid and solid phases have reported both Lachnospiraceae and Ruminococcaceae in higher and in lower abundance in the liquid samples compared with the solid $[19,54]$. These conflicting results could be due to the different diets used in these studies. Animals on all forage diets had higher abundances of both families in liquid phase, while cattle on a diet with a forage to concentrate ratio of 70:30 had lower abundances of these families in the solid phase $[19,54]$. Lower resolution of the taxa might lend clues as to the cause of these differing results.

In agreement with our study, others have found that Prevotellaceae were most abundant in liquid phase compared with solid phase and the dominant family in the liquid fraction 
$622[19,21,22]$. Prevotella sp. are capable of degrading a wide variety of substrates including pectin,

623 hemicellulose, protein, fatty acids, and starch [55]. Readily fermentable carbohydrates including

624 sugars and soluble fiber in the liquid fraction likely support the presence of Prevotella. Thus, the

625 lower abundance of Prevotella in samples with increased solid fraction, including grab samples

626 and solid strained was logical.

627 Our data show that ASVs from Kiritimatiellaeota had significantly higher abundances in

628 liquid strained samples, but these ASVs were not significantly differentially abundant in solid

629 versus grab samples (Fig 1A and 1C). These data are in agreement with a study that found

630 Kiritimatiellaeota in higher proportion in the liquid compared with the solid phase of a yak

631 rumen [56]. Additionally, an order in this phyla, WCHB1-41, was identified to be part of the

632 "core microbiome" in liquid samples from the rumen [57]. Kiritimatiellaeota was found in rumen

633 samples and was in higher abundance from samples of higher methane producers making it a

634 potentially important microbe to understand in order to possibly reduce methane emissions [58].

635 Bioinformatic analysis has hypothesized that this phyla uses sodium for a coupling ion to

636 generate the electrochemical gradient to produce ATP, rather than the typical $\mathrm{H}^{+}$[59]. Therefore,

637 in circumstances when concentrations of $\mathrm{H}^{+}$are relatively lower, as when methane emission are

638 high, this phylum could have a competitive advantage of using sodium as a coupling ion. The

639 role of this rumen microbe has yet to be understood and our data demonstrates that for

640 investigators interested in elucidating the role of this microbe in the rumen ecosystem, samples

641 can be enriched with Kiritimatiellaeota by filtering rumen samples through cheese cloth.

\section{Stomach tube samples}

644 Prevotellaceae, Lachnospiraceae and Ruminococcaceae were the predominate families 
645 regardless of the sampling method [8]. Importantly, these authors made a point to include

646 particles attached to the strainer to capture a representative sample in the rumen. Similarly, in the

647 present study Prevotellaceae and Ruminococcaceae (Fig 5A and 5B) were not significantly

648 different at the family level, while Lachnospiraceae was significantly lower in stomach tube

649 samples (Fig 5C). The lower relative abundance of Lachnospiraceae, specifically the genera

650 Butyrivibrio and Coprococcus, in samples collected by esophageal tube rather than through a

651 rumen fistula was also determined in another study (S2 Fig) [60]. However, at a finer resolution

652 our data showed that these three families had the most significant differentially abundant ASVs

653 when comparing the stomach tube and grab samples.

In agreement with De Menezes et al. who found Fibrobacter and Spirochaetes in the

655 solid fraction, the only assigned genera in the family Fibrobacteraceae, Fibrobacter, was

656 significantly lower in abundance in stomach tube samples compared with grab samples (Fig 1C,

6576 and S2 Fig) as was the family Spirochaetaceae (Fig 1C and 6) due to a lower abundance of the 658 genus Treponema (S2 Fig) [61]. Initially, we hypothesized the lower abundance of Fibrobacter

659 species in stomach tube samples would largely be driven by the exclusion of fibrous particles in 660 the sample as Fibrobacter facilitates cellulose degradation in the rumen [62-64]. However, 661 significantly lower abundances of the family Fibrobacteraceae and Fibrobacter at the genus

662 level were seen in solid and liquid unstrained samples compared to grab samples (Fig 6 and S2

663 Fig). Alternatively, the differences could be attributed to location of rumen sampling.

665 Ruminococcus_1) did follow the expected pattern of significantly lower abundance in stomach

666 tube and liquid samples and significantly higher abundance in solid samples compared with grab

667 samples (S2 Fig). The different distribution of these to cellulolytic species could be reflective of 
their differential preferences for particular plant tissues, for example structural polysaccharides of the cell wall, as a growth substrate [65]. For studies that are interested in fibrolytic bacteria such as Fibrobacter, straining the liquid out of the sample does not enrich for these bacteria, but rather seems to disrupt these communities. Therefore, our data suggests that grab samples are the best option for examining these populations.

An important phylum in defining stomach tube samples was Fusobacteria, which was significantly higher in abundance in stomach tube samples compared with grab samples (Fig 1C). This difference was driven by the genus Fusobacterium (S2 Fig) and to the authors' knowledge this difference between stomach tube and rumen sampling methods has not been previously reported. Fusobacterium necrophorum is an important target species for improving rumen efficiency as it degrades lysine, whose dietary deficiency is the most likely to limit milk production $[66,67]$. In addition, $F$. necrophorum was reported to be an opportunistic pathogen that causes liver abscesses in feedlot cattle $[68,69]$. Our data have identified a previously unreported difference between rumen and stomach tube samples that would enable monitoring of this important genus with stomach tube sampling and has implications for both dairy and beef cattle.

Stomach tube samples more closely reflected liquid samples, but stomach tube samples were highly variable (Fig 3 and S1 Fig). This high variability in microbial community could reflect the fact that the stomach tube did not have a screen, therefore the solid contribution to the stomach tube sample was also highly variable. There were 3,615 ASVs that were in common between stomach tube and grab samples. Two families, Rhodobacteraceae and Spirosomaceae were found in grab, liquid strained and liquid unstrained samples, but were not present in stomach tube samples. However, Solirubrobacteraceae was found only in stomach tube samples. 
691 These differences could reflect differences in the location of the tube placement (cranial ventral)

692

693

694

695

696

697

698

699

700

701

702

703

704

705

706

707

708

709

710

711

712

713

compared with the sampling the rumen from the cannula (central rumen).

Taken together, these data suggest that stomach tube samples could be reflective of rumen samples provided some solid particulate are included and attempts are made to place the tube at a consistent depth. Despite following these precautions, researchers should expect these samples to be more variable than grab samples and increase their sample size accordingly.

\section{Feces vs rumen}

In the current study, as anticipated, fecal samples were not representative of the microbial community of the rumen. The differences between fecal and rumen samples were driven by differences in two Firmicute families: Ruminococcaceae and Lachnospiraceae (Fig 4). Indeed, it was found that there was significantly higher abundance of Ruminococcaceae (Fig 5B) and significantly lower abundance of Lachnospiraceae in feces (Fig 5C). Similarly, Noel et al. found the abundance of Ruminococcaceae to be much higher in feces compared with rumen samples

[70]. However, they found no difference in the abundance of Lachnospiraceae. A recent preprint found strikingly similar relative abundances of top three most abundant families in feces from dairy cattle: Ruminococcaceae (34.9\% compared to our $40.7 \%)$, and Rikenellaceae $(11.6 \%$ compared to our $15.7 \%$ ) and Lachnospiraceae (6.8\% compared to our $7.7 \%)$ [71]. These data show that Ruminococcaceae is typically found in higher abundance in feces, while fecal Lachnospiraceae will have lower abundance than the rumen population.

Both Lachnospiraceae and Ruminococcaceae are also members of the human gastrointestinal tract and have multiple glycoside hydrolases $(\mathrm{GH})$ and carbohydrate-binding modules $(\mathrm{CBM})$ that allow utilization of complex plant material, and transport degradation products of various sizes and compositions [72]. Their differences in abundance between the 
rumen and fecal samples was likely a reflection of their specialization in degrading the various types of substrates present in these two niches. As both families contain butyrate producers, the shift in these families could represent a change in the major sources of butyrate in the rumen compared with the lower colon. The reader should note that there are discrepancies in the literature as to the taxonomy of genera in Lachnospiraceae [73]. Of note is a prominent butyrate producer Eubacterium rectale that is cited as belonging to both Eubacteriaceae and Lachnospiraceae, despite its placement on a 16S rRNA gene tree near recognized members of Lachnospiraceae [74]. These inconsistencies can make appropriate comparisons at the level of family across studies difficult.

In addition, to the families that drove the major differences between rumen and feces, other families were also found to be differentially abundant between these two sample types. There was significantly higher abundance of Akkermansiaceae in feces compared with grab samples (Fig 6B). Until 2016, Akkermansiaceae only contained the species Akkermansia muciniphila, when a novel strain, Akkermansia glycaniphila, was isolated from the feces of a reticulated python [75]. Muciniphila means "mucin-loving" in Latin and as its name suggests $A$. muciniphila is a mucin-degrader, which produces acetate and propionate from mucin fermentation [76]. This species is known to be one of the most abundant in the human colon making up $0.5-5 \%$ of the total bacteria, which was in agreement with the relative abundance we observed (Fig 7B) [77,78]. Other studies have also noted the higher abundances of Akkermansia in feces compared with rumen samples $[28,79]$. In humans, A. muciniphilia had a protective effect against obesity and played a role in both glucose and lipid metabolism [80,81]. Akkermansia also had anti-inflammatory effects that were in part mediated through a membrane specific protein that interacted with the toll-like receptor-2 and improved gut-barrier function 
737 when given orally [82]. Due to the role of $A$. muciniphilia in regulating intestinal inflammation

738 and fat deposition, a better understanding of its function in cattle could identify methods to

739 improve weight gain in cattle.

740 Taken together, fecal samples are not an accurate representation of rumen samples as

741 they have differences in the abundance of predominant families in the phyla Firmicutes and

742 Bacteroidetes. Fecal samples differed from those taken from the rumen as they had significantly

743 lower relative abundance of Lachnospiraceae, Christensenellaceae, Prevotellaceae, Fibrobacter

744 and Treponema (Fig 5A and C, 6, 7 and S2 Fig). Also, fecal samples had significantly higher

745 relative abundance of Ruminococcaceae, Rikenellaceae and Akkermansia compared with grab

746 samples (Fig 5B, 6, 7 and S2 Fig). Researchers can access the freely accessible data found at

747 https://doi.org/10.5281/zenodo.4026849 to determine how sampling methods might affect the

748 abundance of their microbe of interest.

749 Archaeal populations

750 Feces vs rumen

Methanogens are an important functional group within the rumen as their use of $\mathrm{H}_{2}$ to

752 reduce $\mathrm{CO}_{2}$ to methane $\left(\mathrm{CH}_{4}\right)$ removes $\mathrm{H}_{2}$ from the rumen that is generated during fermentation

753 of carbohydrates [83,84]. Methane has a global warming potential 28-34 fold higher than $\mathrm{CO}_{2}$

754 over 100 years, and therefore its mitigation is important to reducing the environmental impact of

755 animal agriculture. Additionally, methane production is energy inefficient, resulting in a $2-12 \%$

756 loss in gross energy intake in cattle [85]. There is very limited data on differences between the

757 archaeal populations in the rumen compared with the feces, as a majority of studies solely focus

758 on the rumen population. 
One study that has examined both the rumen and fecal populations of archaea of Nelore

760

761

762

763

764

765

766

767

768

769

770

771

772

773

774

775

776

777

778

779

780

781

cattle was conducted by Andrade et al. [50]. Like this present study, Andrade et al. also utilized DADA2 to identify ASVs and assigned taxa with the SILVA database v132; however, they used different primers that are specific for archaea and bacteria rather than universal primers and classified archaeal sequences using the Rumen and Intestinal Methanogen database (RIM-DB).

Together these choices allowed Andrade et al. to classify archaeal ASVs down to the species level, which contrasted with this present study where methanogenic ASVs were only classified down to the genus level. Other than Methanobrevibacter and Methanosphaera, the other archaeal genera that this present study and Andrade et al. identified were different. Our data contained Methanocorpusculum, Methanimicrococcus and Candidatus Methanomethylophilus while Andrade et al. observed Methanomicrobium. Both studies found that Methanobrevibacter and Methanosphaera were found in both the rumen and feces; however, there were differences in the relative abundances of the main genera. In contrast to Andrade et al. we found significantly lower relative abundance of Methanobrevibacter in fecal samples compared with samples from the rumen. Despite using similar methods there is not clear agreement as to the differences in abundance of genera and which genera are present in the two populations.

As an alternative to $16 \mathrm{~S}$ rRNA gene sequencing, the mcrA gene can be sequenced to study methanogens $[86,87]$. The mcrA gene encodes the $\alpha$-subunit of the methyl coenzyme M reductase, which catalyzes the last step of methanogenesis and is conserved among all methanogens [88]. A study that used mcrA amplicon sequencing found that the most abundant genera in manure was Methanocorpusculum while in the rumen it was Methanobrevibacter [89]. While we found Methanocorpusculum in our fecal samples it was a minor genus and the discrepancy is most likely explained by differences in the gene amplicon sequences. Taken 
782 together these data suggest Methanobrevibacter is a dominant archaeal genus in both the rumen

783

784

785

786

787

788

789

790

791

792

793

794

795

796

797

798

799

800

801

802

803

804

and fecal populations. The lack of data comparing the rumen and fecal populations suggest that further research is required to understand the archaeal populations.

\section{Rumen samples}

In the present study relative abundance of archaeal families was similar across rumen samples, both liquid and solid phases, with wide variation in the relative abundance of Methanocorpusculaceae (Fig 6). In contrast, Bowen et al. found methanogens to be more abundant in the solid phase [19]. Our data more closely agree with de Mulder et al. who found similar abundance in samples including solid, rumen liquid, and liquid [54]. When we examined the archaeal ASVs in our data at the genus level, Methanosphera was significantly lower in relative abundance in liquid samples compared with grab samples. This is in agreement with previous studies that found Methanosphera was more abundant in the solid phase, rather than the liquid phase $[19,54]$. As a whole these data suggest that the collective abundance of methanogens was similar between solid and liquid phases, but that Methanosphera are found at higher abundance in the rumen liquid. Studies evaluating feed additives or diet alterations to modulate methanogen populations in the rumen should consider including the liquid fraction of rumen fluid to capture changes in the abundance of Methanosphera.

At the family level three methanogenic families, Methanomethylophilaceae, Methanobacteriaceae and Methanocorpusculaceae, were not significantly different between the grab sample and samples acquired via a stomach tube (Fig 6). However, there were 4 ASVs assigned to Methanobrevibacter that were found to be significantly higher in abundance in stomach tube samples. This is a paradoxical finding as stomach tube samples typically have 
more liquid than solid particles in them and we previously noted that Methanosphaera was in higher abundance in liquid samples. As the coefficient for the difference in relative abundance of Methanobrevibacter is low (0.1-0.5), we believe that in practice with higher numbers of animals this difference would be negligible.

Many of the differences described thus far have focused on the major genera Methanobrevibacter and Methanosphaera, which are hydrogenotrophic methanogens. While the hydrogenotrophic pathway for methane production is the most common there are two alternative pathways: methylotrophic and acetoclastic that utilize methylated compounds and acetate, respectively. Thus far, only taxa within the order Methanosarcinales have been identified to be capable of acetoclastic methanogenesis [90,91]. An acetoclastic methanogen in our data, Methanimicrococcus, was only present in two liquid samples. There was not a strong pattern as to the phase in which this minor genus may be found, and as deep sequencing would be required to determine shifts in its abundance, targeted qRT-PCR would be a better choice to study abundance of this microbe. In addition, there was one ASV assigned to Methnomethylophilaceae, a methylotrophic archaeon, that was significantly higher in abundance in stomach tube samples compared with grab samples, although at higher taxonomic levels no differences were found for the family Methnomethylophilaceae.

Taken together these results demonstrate that stomach tubing would likely provide a representative community of major populations of methanogens, Methanosphaera and Methanobrevibacter, compared with grab samples. For minor populations accurate surveys would require more targeted techniques, such as qRT-PCR or mcrA sequencing. While this study added to an understanding of how sampling methods will potentially impact archaea populations observed, it should not be considered a comprehensive evaluation of the microbial communities. 
Specific archaeal primers and qRT-PCR could be used to clarify discrepancies between this study and past work. However, for those evaluating archaeal communities with 16S rRNA gene sequencing, this study can serve as a guide to help in study design to improve the chances of capturing an accurate picture of the taxa of interest.

\section{Acknowledgements}

We thank the UC Davis DNA Technologies Core for sequencing services. We appreciate the work of the UC Davis Dairy manager Douglas Gisi and Carlyn Peterson that helped during sample collection.

\section{Funding information}

This work was supported by a multistate project \#NC2042 JH was supported by the Leland Roy Saxon and Georgia Wood Saxon fellowship.

\section{Author contribution statement}

JH analyzed, interpreted data, and wrote manuscript. ML collected samples, prepared libraries, aided in experimental design and edited manuscript. ED designed the experiment, assisted in sample collection helped prepared manuscript. EAM edited manuscript.

\section{Conflict of interest statement}

The authors have not conflicts of interest to declare.

\section{Supporting information}

\section{S1 Fig. Double principal coordiant analysis of the Bray-Curtis distance after removal of}

the phyla Bacteroidetes and Firmicutes from the dataset. DPCoA is a phylogenetic

ordination method and that provides a biplot representation of both (A) samples and (B) 
taxonomic categories. The $1^{\text {st }}$ axis separtes liquid strained samples from other rumen sample types while the $2^{\text {nd }}$ axis discrimates fecal from rumen samples. Samples that have larger scores on the $1^{\text {st }}$ axis have more taxa from the phylum Kiritimatiellaeota and less taxa from the phylum Euryarchaeota. Likewise, samples with higher scores on the $2^{\text {nd }}$ axis have more taxa from the family Akkermansiaceae and less taxa from the families Fibrobacteraceae and Spirochaetaceae. To faithfully reflect the variance in the coordinates, the height-to-width ratio was based on the ratio between the corresponding eigenvalues.

\section{S2 Fig. Significant genera that are differentially abundant across sample type graph as} coefficients with a $95 \%$ confidence interval calculated from the corncob model. Taxa with negative coefficients for a sample type are expected to have a lower relative abundance when compared to the grab samples while positive coefficients suggest a higher relative abundance in that sample type compared to grab samples. Taxa are presented with phylum, family, genus and species to the lowest assigned level.

\section{References}

1. Stewart CS, Flint HJ, Bryant MP. The Rumen Bacteria. 2nd ed. In: Hobson PN, Stewart CS, editors. The Rumen Microbial Ecosystem. 2nd ed. London: Blackie Academic \& Professional; 1997. pp. 10-72.

2. Krehbiel CR. INVITED REVIEW: Applied nutrition of ruminants: Fermentation and digestive physiology. Prof Anim Sci. 2014;30: 129-139. doi:10.15232/S1080$7446(15) 30100-5$

3. Jami E, White BA, Mizrahi I. Potential role of the bovine rumen microbiome in modulating milk composition and feed efficiency. PLoS One. 2014;9. doi:10.1371/journal.pone.0085423 
872 4. Hernandez-Sanabria E, Goonewardene LA, Wang Z, Durunna ON, Moore SS, Guan LL.

873

874

875

876

877

878

879

880

881

882

883

884

885

886

887

888

889

890

891

892

893

894

Impact of feed efficiency and diet on adaptive variations in the bacterial community in the rumen fluid of cattle. Appl Environ Microbiol. 2012;78: 1203-1214.

doi:10.1128/AEM.05114-11

5. Liu C, Meng Q, Chen Y, Xu M, Shen M, Gao R, et al. Role of age-related shifts in rumen bacteria and methanogens in methane production in cattle. Front Microbiol. 2017;8: 1-14. doi:10.3389/fmicb.2017.01563

6. Guan LL, Nkrumah JD, Basarab JA, Moore SS. Linkage of microbial ecology to phenotype: correlation of rumen microbial ecology to cattle's feed efficiency. FEMS Microbiol Lett. 2008;288.

7. Fan P, Bian B, Teng L, Nelson CD, Driver J, Elzo MA, et al. Host genetic effects upon the early gut microbiota in a bovine model with graduated spectrum of genetic variation.

ISME J. 2020;14: 302-317. doi:10.1038/s41396-019-0529-2

8. Paz HA, Anderson CL, Muller MJ, Kononoff PJ, Fernando SC. Rumen Bacterial Community Composition in Holstein and Jersey Cows Is Different under Same Dietary Condition and Is Not Affected by Sampling Method. Front Microbiol. 2016;7: 1-9. doi:10.3389/fmicb.2016.01206

9. Noel SJ, Attwood GT, Rakonjac J, Moon CD, Waghorn GC, Janssen PH. Seasonal changes in the digesta-adherent rumen bacterial communities of dairy cattle grazing pasture. PLoS One. 2017;12: 1-18. doi:10.1371/journal.pone.0173819

10. Petri RM, Forster RJ, Yang W, McKinnon JJ, McAllister T a. Characterization of rumen bacterial diversity and fermentation parameters in concentrate fed cattle with and without forage. J Appl Microbiol. 2012;112: 1152-1162. doi:10.1111/j.1365-2672.2012.05295.X 
895 11. Zhang J, Shi H, Wang Y, Li S, Cao Z, Ji S, et al. Effect of dietary forage to concentrate ratios on dynamic profile changes and interactions of ruminal microbiota and metabolites in holstein heifers. Front Microbiol. 2017;8: 1-18. doi:10.3389/fmicb.2017.02206

12. Dehority BA, Orpin CG. Development of, and natural fluctuations in, rumen microbial populations. In: Hobson PN, Stewart CS, editors. The Rumen Microbial Ecosystem. London, UK: Blackie Academic \& Professional; 1997. pp. 196-245.

901 13. Kamra DN. Rumen microbial ecosystem. Curr Sci. 2005;89: 124-135.

902 14. Wolin MJ, Miller HJ. Microbe-Microbe Interactions. 2nd ed. In: Hobson PN, Stewart CS, 903 editors. The Rumen Microbial Ecosystem. 2nd ed. London, UK: Blackie Academic \& Professional; 1997. pp. 467-491.

15. Hungate RE. The Rumen and Its Microbes. The Rumen and its Microbes. New

907 16. Xue M, Sun H, Wu X, Guan LL, Liu J. Assessment of rumen microbiota from a large dairy cattle cohort reveals the pan and core bacteriomes contributing to varied phenotypes. Appl Environ Microbiol. 2018;84: 1-13. doi:10.1128/AEM.00970-18

17. Wu S, Baldwin RL, Li W, Li C, Connor EE, Li RW. The Bacterial Community Metagenomics. 2012;1: 1-11. doi:10.4303/mg/235571

913 18. Steiner S, Neidl A, Linhart N, Tichy A, Gasteiner J, Gallob K, et al. Randomised prospective study compares efficacy of five different stomach tubes for rumen fluid sampling in dairy cows. Vet Rec. 2015;176: 50. doi:10.1136/vr.102399 communities associated with the liquid and solid phases of the rumen of cattle offered a 
diet of perennial ryegrass or white clover. Front Microbiol. 2018;9: 1-8. doi:10.3389/fmicb.2018.02389

920

921

922

923

924

925

926

927

928

929

930

931

932

933

934

935

936

937

938

939

940

20. Cho SJ, Cho KM, Shin EC, Lim WJ, Hong SY, Choi BR, et al. 16S rDNA analysis of bacterial diversity in three fractions of cow rumen. J Microbiol Biotechnol. 2006;16: 92101.

21. Pitta DW, Pinchak WE, Dowd SE, Osterstock J, Gontcharova V, Youn E, et al. Rumen bacterial diversity dynamics associated with changing from bermudagrass hay to grazed winter wheat diets. Microb Ecol. 2010;59: 511-522. doi:10.1007/s00248-009-9609-6

22. Schären M, Kiri K, Riede S, Gardener M, Meyer U, Hummel J, et al. Alterations in the rumen liquid-, particle- and epithelium-associated microbiota of dairy cows during the transition from a silage- and concentrate-based ration to pasture in spring. Front Microbiol. 2017;8. doi:10.3389/fmicb.2017.00744

23. Lengowski MB, Witzig M, Möhring J, Seyfang GM, Rodehutscord M. Effects of corn silage and grass silage in ruminant rations on diurnal changes of microbial populations in the rumen of dairy cows. Anaerobe. 2016;42: 6-16. doi:10.1016/j.anaerobe.2016.07.004

24. McCann JC, Luan S, Cardoso FC, Derakhshani H, Khafipour E, Loor JJ. Induction of subacute ruminal acidosis affects the ruminal microbiome and epithelium. Front Microbiol. 2016;7: 1-18. doi:10.3389/fmicb.2016.00701

25. Ramos-Morales E, Arco-Pérez A, Martín-García AI, Yáñez-Ruiz DR, Frutos P, Hervás G. Use of stomach tubing as an alternative to rumen cannulation to study ruminal fermentation and microbiota in sheep and goats. Anim Feed Sci Technol. 2014;198: 5766. doi:10.1016/j.anifeedsci.2014.09.016

26. Lodge-Ivey SL, Browne-Silva J, Horvath MB. Technical note: Bacterial diversity and 
fermentation end products in rumen fluid samples collected via oral lavage or rumen cannula. J Anim Sci. 2009;87: 2333-2337. doi:10.2527/jas.2008-1472

27. Duffield T, Plaizier JC, Fairfield A, Bagg R, Vessie G, Dick P, et al. Comparison of techniques for measurement of rumen $\mathrm{pH}$ in lactating dairy cows. J Dairy Sci. 2004;87: 59-66. doi:10.3168/jds.S0022-0302(04)73142-2

28. Liu J hua, Zhang M ling, Zhang R yang, Zhu W yun, Mao S yong. Comparative studies of the composition of bacterial microbiota associated with the ruminal content, ruminal epithelium and in the faeces of lactating dairy cows. Microb Biotechnol. 2016;9: 257-268. doi:10.1111/1751-7915.12345

29. Mohammadzadeh H, Yáñez-Ruiz DR, Martínez-Fernandez G, Abecia L. Molecular comparative assessment of the microbial ecosystem in rumen and faeces of goats fed alfalfa hay alone or combined with oats. Anaerobe. 2014;29: 52-58. doi:10.1016/j.anaerobe.2013.11.012

30. Derakhshani H, Tun HM, Cardoso FC, Plaizier JC, Khafipour E, Loor JJ. Linking Peripartal Dynamics of Ruminal Microbiota to Dietary Changes and Production Parameters. Front Microbiol. 2017;7: 1-13. doi:10.3389/fmicb.2016.02143

31. Weimer PJ, Cox MS, Vieira de Paula T, Lin M, Hall MB, Suen G. Transient changes in milk production efficiency and bacterial community composition resulting from near-total exchange of ruminal contents between high- and low-efficiency Holstein cows. J Dairy Sci. 2017;100: 7165-7182. doi:10.3168/jds.2017-12746

32. Mon KKZ, Saelao P, Halstead MM, Chanthavixay G, Chang H-C, Garas L, et al. Salmonella enterica Serovars Enteritidis Infection Alters the Indigenous Microbiota Diversity in Young Layer Chicks. Front Vet Sci. 2015;2: 61. 
33. McIver LJ, Abu-Ali G, Franzosa EA, Schwager R, Morgan XC, Waldron L, et al.

BioBakery: A meta’omic analysis environment. Bioinformatics. 2018;34: 1235-1237.

34. Martin M. Cutadapt removes adapter sequences from high-throughput sequencing reads.

35. Callahan BJ, McMurdie PJ, Rosen MJ, Han AW, Johnson AJA, Holmes SP. DADA2:

36. Caporaso JG, Kuczynski J, Stombaugh J, Bittinger K, Bushman FD, Costello EK, et al. QIIME allows analysis of high-throughput community sequencing data. Nat Methods.

37. McMurdie PJ, Holmes S. Phyloseq: an R package for reproducible interactive analysis and graphics of microbiome census data. PLoS One. 2013;8: e61217.

38. Pavoine S, Dufour AB, Chessel D. From dissimilarities among species to dissimilarities among communities: A double principal coordinate analysis. J Theor Biol. 2004;228: 523-537. doi:10.1016/j.jtbi.2004.02.014

39. Martin BD, Witten D, Willis AD. Modeling microbial abundances and dysbiosis with beta-binomial regression. Ann Appl Stat. 2020;14: 94-115. doi:10.1214/19-AOAS1283

40. Willis AD, Martin BD. Estimating diversity in networked ecological communities. 
1049. doi:10.1111/biom. 12332

988

989

990

991

992

993

994

995

996

997

998

999

1000

1001

1002

1003

1004

1005

1006

1007

1008

1009

42. Willis A, Bunge J, Whitman T. Improved detection of changes in species richness in high diversity microbial communities. J R Stat Soc Ser C Appl Stat. 2017;66: 963-977. doi:10.1111/rssc. 12206

43. Tibshirani R, Walther G, Hastie T. Estimating the number of clusters in a data set via the gap statistic. J R Stat Soc Ser B Stat Methodol. 2001;63: 411-423. doi:10.1111/14679868.00293

44. Callahan BJ, McMurdie PJ, Holmes SP. Exact sequence variants should replace operational taxonomic units in marker-gene data analysis. ISME J. 2017;11: 2639-2643. doi:10.1038/ismej.2017.119

45. Kim M, Morrison M, Yu Z. Status of the phylogenetic diversity census of ruminal microbiomes. FEMS Microbiol Ecol. 2011;76: 49-63. doi:10.1111/j.15746941.2010.01029.x

46. Wong K, Shaw TI, Oladeinde A, Glenn TC, Oakley B, Molina M. Rapid microbiome changes in freshly deposited cow feces under field conditions. Front Microbiol. 2016;7: 112. doi:10.3389/fmicb.2016.00500

47. Skarlupka JH, Kamenetsky ME, Jewell KA, Suen G. The ruminal bacterial community in lactating dairy cows has limited variation on a day-to-day basis. J Anim Sci Biotechnol. 2019;10: 1-5. doi:10.1186/s40104-019-0375-0

48. Bainbridge ML, Cersosimo LM, Wright ADG, Kraft J. Rumen bacterial communities shift across a lactation in Holstein, Jersey and Holstein $\times$ Jersey dairy cows and correlate to rumen function, bacterial fatty acid composition and production parameters. FEMS Microbiol Ecol. 2016;92: 1-14. doi:10.1093/femsec/fiw059 
1010 49. Lourenco JM, Kieran TJ, Seidel DS, Glenn TC, Da Silveira MF, Callaway TR, et al.

1011

1012

1013

1014

1015

1016

1017

1018

1019

1020

1021

1022

1023

1024

1025

1026

1027

1028

1029

1030

1031

1032

Comparison of the ruminal and fecal microbiotas in beef calves supplemented or not with concentrate. PLoS One. 2020;15: 1-17. doi:10.1371/journal.pone.0231533

50. Andrade BGN, Bressani FA, Cuadrat RRC, Tizioto PC, De Oliveira PSN, Mourão GB, et al. The structure of microbial populations in Nelore GIT reveals inter-dependency of methanogens in feces and rumen. J Anim Sci Biotechnol. 2020;11: 1-10. doi:10.1186/s40104-019-0422-x

51. Ji S, Zhang H, Yan H, Azarfar A, Shi H, Alugongo G, et al. Comparison of rumen bacteria distribution in original rumen digesta, rumen liquid and solid fractions in lactating Holstein cows. J Anim Sci Biotechnol. 2017;8: 1-7. doi:10.1186/s40104-017-0142-z

52. Jewell KA, McCormick CA, Odt CL, Weimer PJ, Suen G. Ruminal bacterial community composition in dairy cows is dynamic over the course of two lactations and correlates with feed efficiency. Appl Environ Microbiol. 2015;81: 4697-4710. doi:10.1128/AEM.0072015

53. Willis AD. Rarefaction, alpha diversity, and statistics. Front Microbiol. 2019;10. doi:10.3389/fmicb.2019.02407

54. De Mulder T, Goossens K, Peiren N, Vandaele L, Haegeman A, De Tender C, et al. Exploring the methanogen and bacterial communities of rumen environments: solid adherent, fluid and epimural. FEMS Microbiol Ecol. 2017;93: 1-12. doi:10.1093/femsec/fiw251

55. Russell JB. Rumen Microbiology and its Role in Ruminant Nutrition. Ithaca. New York: Dept. of Microbiology, Cornell University; 2002.

56. Ren Q, Si H, Yan X, Liu C, Ding L, Long R, et al. Bacterial communities in the solid, 
liquid, dorsal, and ventral epithelium fractions of yak (Bos grunniens) rumen.

1034

1035

1036

1037

1038

1039

1040

1041

1042

1043

1044

1045

1046

1047

1048

1049

1050

1051

1052

1053

1054

1055

Microbiologyopen. 2020;9: 1-16. doi:10.1002/mbo3.963

57. Wallace JR, Sasson G, Garnsworthy PC, Tapio I, Gregson E, Bani P, et al. A heritable subset of the core rumen microbiome dictates dairy cow productivity and emissions. Sci Adv. 2019;5. doi:10.1126/sciadv.aav8391

58. Auffret MD, Stewart R, Dewhurst RJ, Duthie CA, Rooke JA, Wallace RJ, et al. Identification, comparison, and validation of robust rumen microbial biomarkers for methane emissions using diverse Bos Taurus breeds and basal diets. Front Microbiol. 2018;8: 1-15. doi:10.3389/fmicb.2017.02642

59. Spring S, Bunk B, Spröer C, Schumann P, Rohde M, Tindall BJ, et al. Characterization of the first cultured representative of Verrucomicrobia subdivision 5 indicates the proposal of a novel phylum. ISME J. 2016;10: 2801-2816. doi:10.1038/ismej.2016.84

60. Henderson G, Cox F, Kittelmann S, Miri VH, Zethof M, Noel SJ, et al. Effect of DNA extraction methods and sampling techniques on the apparent structure of cow and sheep rumen microbial communities. PLoS One. 2013;8: 1-14. doi:10.1371/journal.pone.0074787

61. De Menezes AB, Lewis E, O’Donovan M, O’Neill BF, Clipson N, Doyle EM. Microbiome analysis of dairy cows fed pasture or total mixed ration diets. FEMS Microbiol Ecol. 2011;78: 256-265. doi:10.1111/j.1574-6941.2011.01151.x

62. Flint HJ. The rumen microbial ecosystem—some recent developments. Trends Microbiol. 1997;5: 483-488. doi:10.1016/S0966-842X(97)01159-1

63. Hungate RE. The Rumen Microbial Ecosystem. Annual Review of Ecology and Systematics. 1975. doi:10.1146/annurev.es.06.110175.000351 
1056

1057

1058

1059

1060

1061

1062

1063

1064

1065

1066

1067

1068

1069

1070

1071

1072

1073

1074

1075

1076

1077

1078

64. Dehority BA, Scott HW. Extent of Cellulose and Hemicellulose Digestion in Various Forages by Pure Cultures of Rumen Bacteria. J Dairy Sci. 1967;50: 1136-1141. doi:10.3168/jds.S0022-0302(67)87579-9

65. Shinkai T, Kobayashi Y. Localization of ruminal cellulolytic bacteria on plant fibrous materials as determined by fluorescence in situ hybridization and real-time PCR. Appl Environ Microbiol. 2007;73: 1646-1652. doi:10.1128/AEM.01896-06

66. Polan CE, Cummins KA, Sniffen CJ, Muscato T V., Vicini JL, Crooker BA, et al. Responses of dairy cows to supplemental rumen-protected forms of methionine and lysine. J Dairy Sci. 1991;74: 2997-3013. doi:10.3168/jds.S0022-0302(91)78486-5

67. Russell JB. Factors affecting lysine degradation by ruminal fusobacteria. FEMS Microbiol Ecol. 2006;56: 18-24. doi:10.1111/j.1574-6941.2006.00041.x

68. Tadepalli S, Narayanan SK, Stewart GC, Chengappa MM, Nagaraja TG. Fusobacterium necrophorum: A ruminal bacterium that invades liver to cause abscesses in cattle.

Anaerobe. 2009;15: 36-43. doi:10.1016/j.anaerobe.2008.05.005

69. Nagaraja TG, Chengappa MM. Liver abscesses in feedlot cattle: a review. J Anim Sci. 1998;76: 287-298. doi:10.2527/1998.761287x

70. Noel SJ, Olijhoek DW, McLean F, Løvendahl P, Lund P, Højberg O. Rumen and fecal microbial community structure of holstein and Jersey dairy cows as affected by breed, diet, and residual feed intake. Animals. 2019;9. doi:10.3390/ani9080498

71. Zhao L, Li X, Atwill ER, Aly S, Williams DR. Dynamic changes in fecal bacterial microbiota of dairy cattle across the production line. bioRxiv. 2020; $1-25$. doi:10.1101/2020.02.21.960500

72. Biddle A, Stewart L, Blanchard J, Leschine S. Untangling the genetic basis of fibrolytic 
specialization by lachnospiraceae and ruminococcaceae in diverse gut communities.

Diversity. 2013;5: 627-640. doi:10.3390/d5030627

1081 73. Haas KN. Expansion of and reclassification within the family Lachnospiraceae. University $1082 \quad$ of Massachusetts Amherst. 2016.

1083 74. Meehan CJ, Beiko RG. A phylogenomic view of ecological specialization in the 1084 lachnospiraceae, a family of digestive tract-associated bacteria. Genome Biol Evol. 2014;6: 703-713. doi:10.1093/gbe/evu050

75. Ouwerkerk JP, Aalvink S, Belzer C, de Vos WM. Akkermansia glycaniphila sp. nov., an anaerobic mucin-degrading bacterium isolated from reticulated python faeces. Int J Syst Evol Microbiol. 2016;66: 4614-4620. doi:10.1099/ijsem.0.001399

76. Derrien M, Vaughan EE, Plugge CM, de Vos WM. Akkermansia municiphila gen. nov., sp. nov., a human intestinal mucin-degrading bacterium. Int J Syst Evol Microbiol. 2004;54: 1469-1476. doi:10.1099/ijs.0.02873-0

77. Collado MC, Derrien M, Isolauri E, De Vos WM, Salminen S. Intestinal integrity and Akkermansia muciniphila, a mucin-degrading member of the intestinal microbiota present in infants, adults, and the elderly. Appl Environ Microbiol. 2007;73: 7767-7770. doi:10.1128/AEM.01477-07

78. Derrien M, Collado MC, Ben-Amor K, Salminen S, De Vos WM. The mucin degrader Akkermansia muciniphila is an abundant resident of the human intestinal tract. Appl Environ Microbiol. 2008;74: 1646-1648. doi:10.1128/AEM.01226-07 
pyrosequencing. J Anim Sci. 2010;88: 3977-3983. doi:10.2527/jas.2010-2900

1103 80. Cani PD, de Vos WM. Next-generation beneficial microbes: The case of Akkermansia muciniphila. Front Microbiol. 2017;8: 1-8. doi:10.3389/fmicb.2017.01765

1105 81. Xu Y, Wang N, Tan HY, Li S, Zhang C, Feng Y. Function of Akkermansia muciniphila in obesity: Interactions with lipid metabolism, immune response and gut systems. Front Microbiol. 2020;11: 1-12. doi:10.3389/fmicb.2020.00219

82. Plovier H, Everard A, Druart C, Depommier C, Van Hul M, Geurts L, et al. A purified Possibilities of Interventions. Front Microbiol. 2020;11. doi:10.3389/fmicb.2020.00589

84. Janssen PH. Influence of hydrogen on rumen methane formation and fermentation balances through microbial growth kinetics and fermentation thermodynamics. Anim Feed

Sci Technol. 2010;160: 1-22. doi:10.1016/j.anifeedsci.2010.07.002

85. Johnson KA, Johnson DE. Methane emissions from cattle. J Anim Sci. 1995;73: 24832492. doi: $10.2527 / 1995.7382483 x$ in the phylogenetic analysis of methanogen populations in landfill. Microbiology. 2002;148: 3521-3530. doi:10.1099/00221287-148-11-3521

1124 88. Friedrich MW. Methyl-coenzyme M reductase genes: Unique functional markers for 
methanogenic and anaerobic methane-oxidizing Archaea. Methods Enzymol. 2005;397: 428-442. doi:10.1016/S0076-6879(05)97026-2

1127 89. Ozbayram E, Ince O, Ince B, Harms H, Kleinsteuber S. Comparison of rumen and manure microbiomes and implications for the inoculation of anaerobic digesters. Microorganisms. 2018;6: 15. doi:10.3390/microorganisms6010015

1130 90. Lang K, Schuldes J, Klingl A, Poehlein A, Daniel R, Brune A. New mode of energy metabolism in the seventh order of methanogens as revealed by comparative genome analysis of "Candidatus Methanoplasma termitum.” Appl Environ Microbiol. 2015;81: 1338-1352. doi:10.1128/AEM.03389-14

1134 91. Fournier GP, Gogarten JP. Evolution of acetoclastic methanogenesis in Methanosarcina 1135 via horizontal gene transfer from cellulolytic Clostridia. J Bacteriol. 2008;190: 1124 1127. doi:10.1128/JB.01382-07 
Bacteroidetes

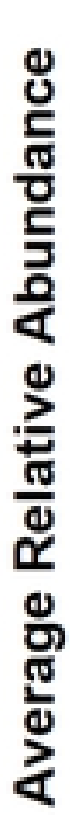
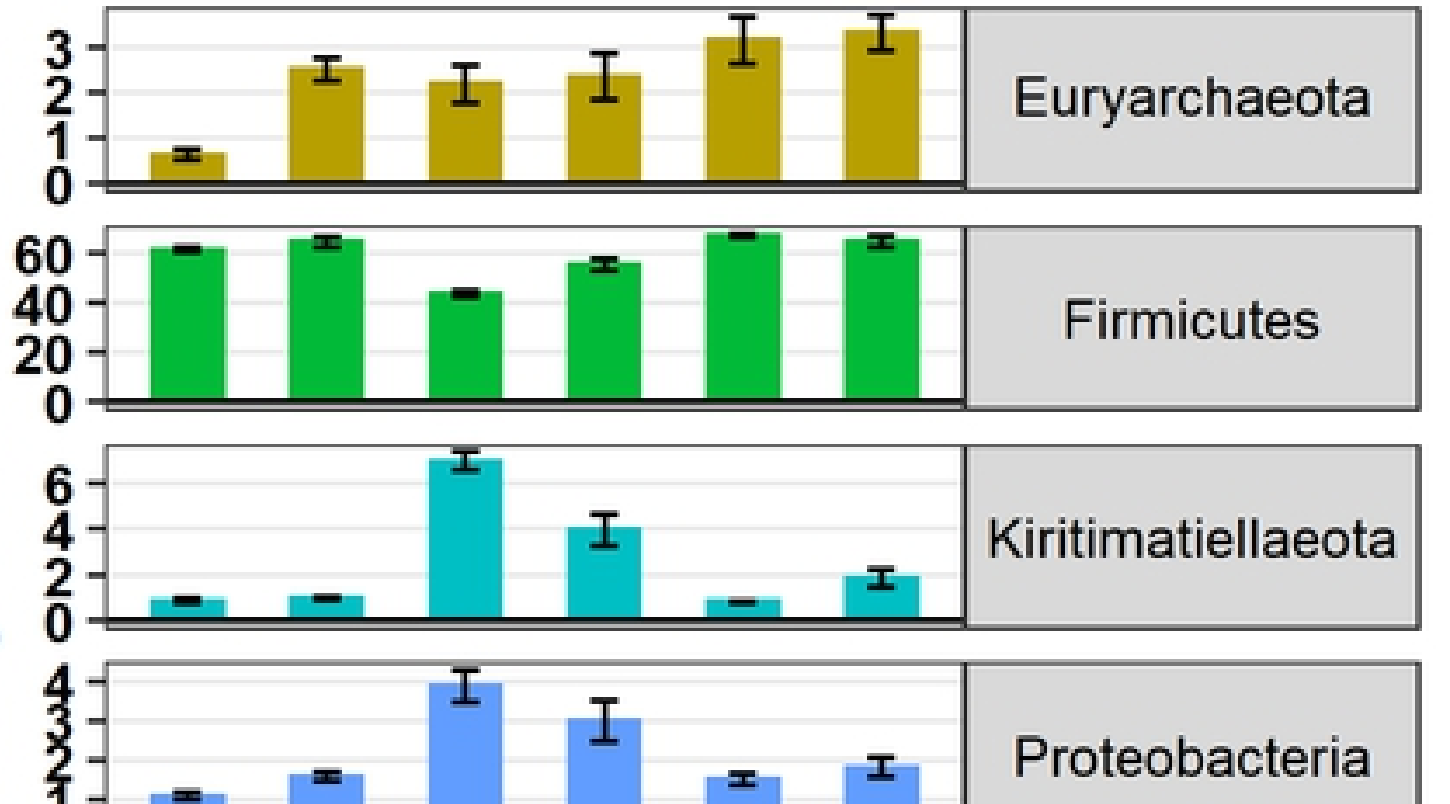

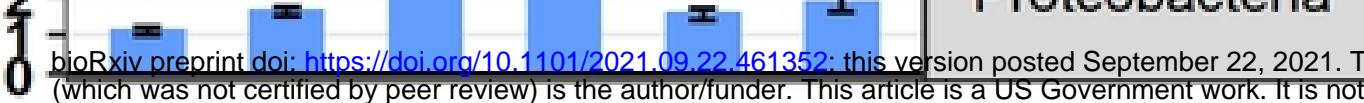
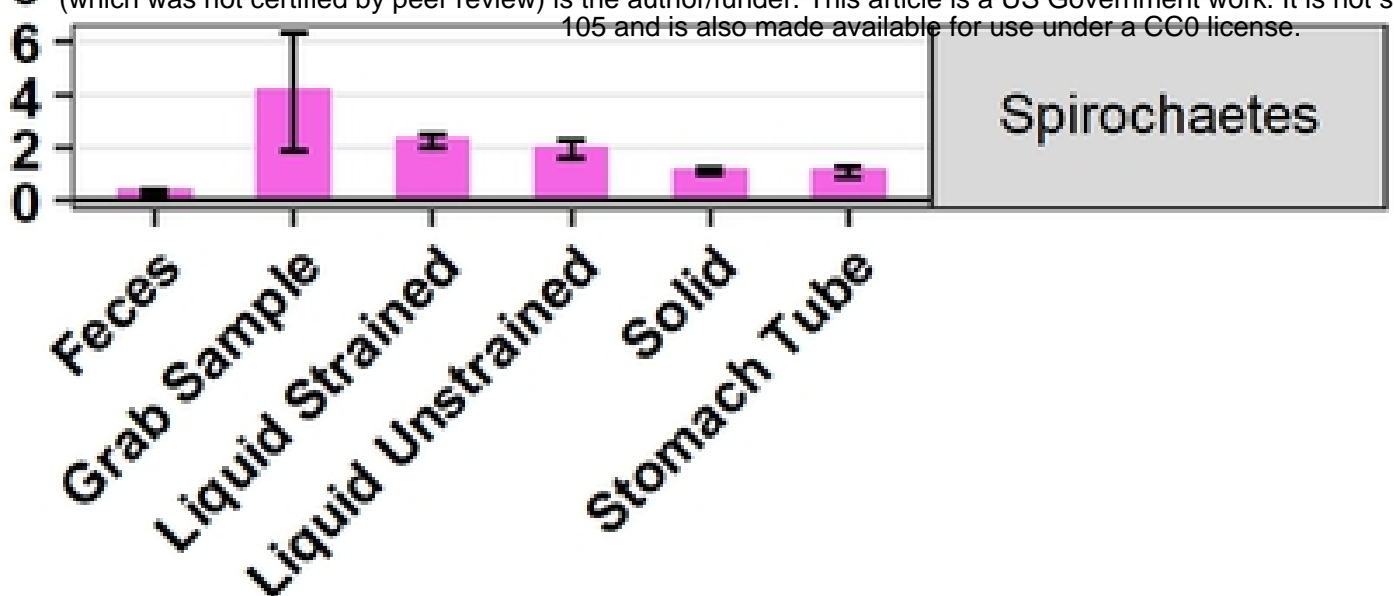

C
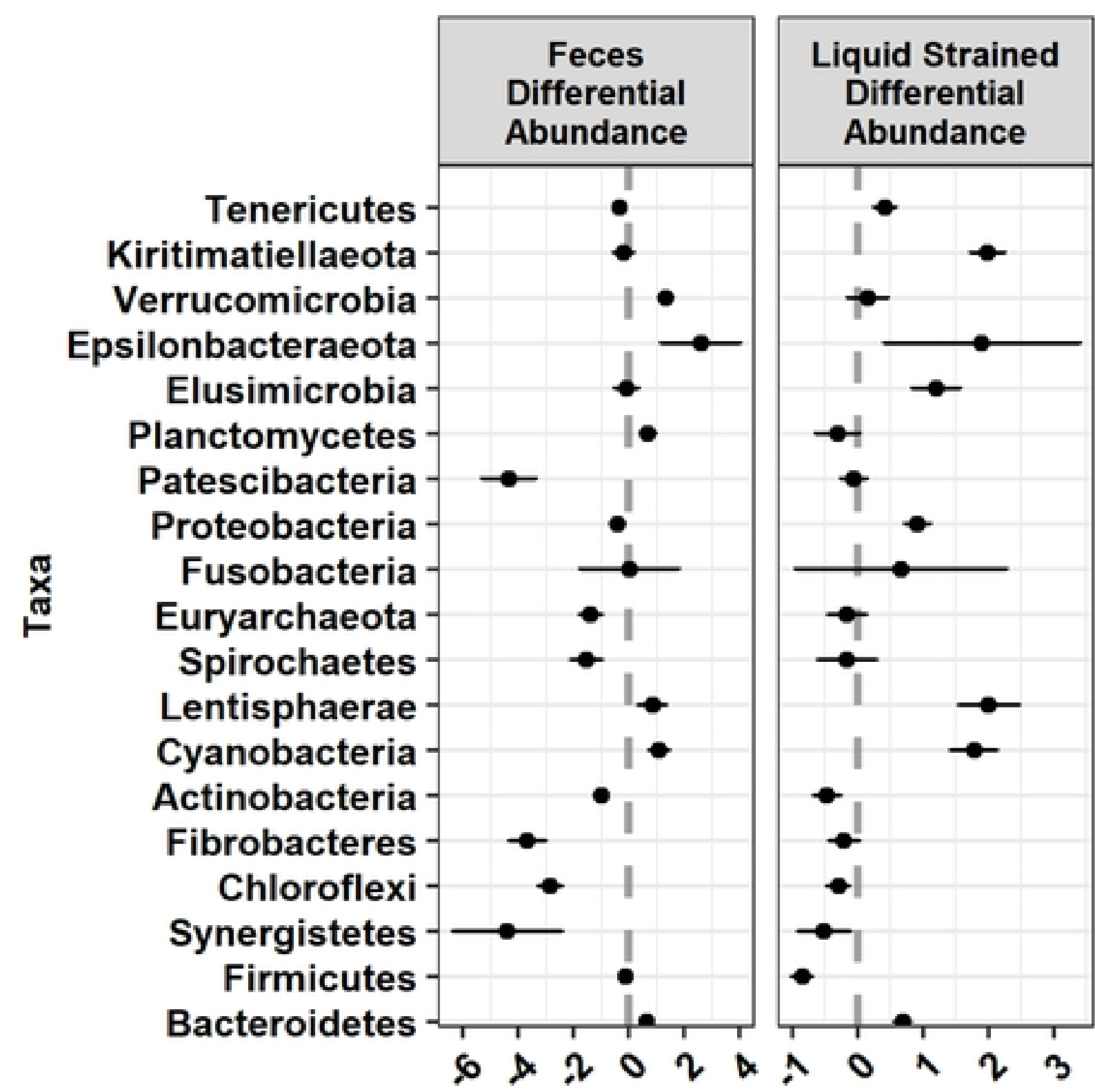

B

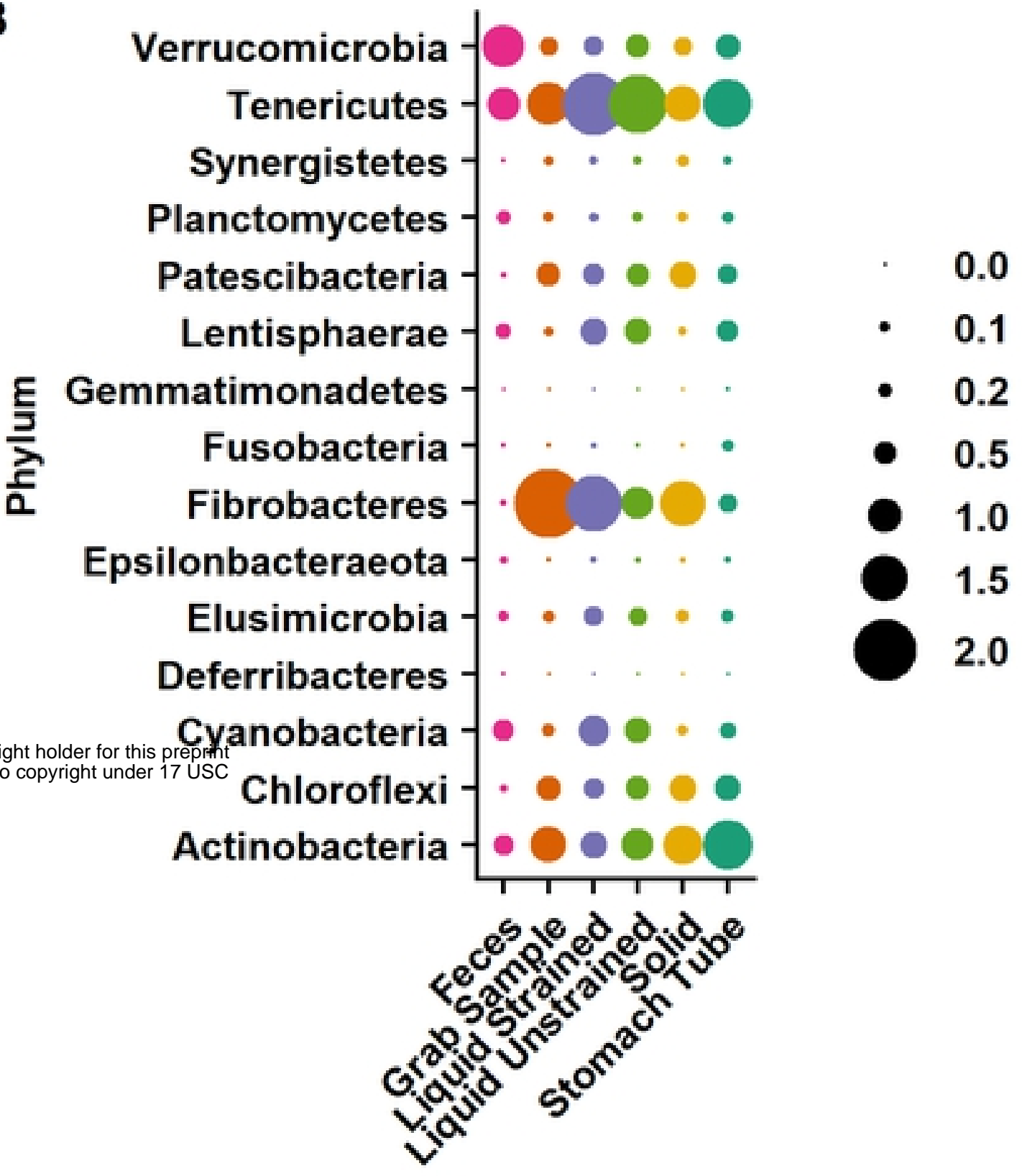

Synergistetes

Planctomycetes

Patescibacteria

0.2

0.5

1.0

1.5

2.0
Cyanobacteria

Actinobacteria
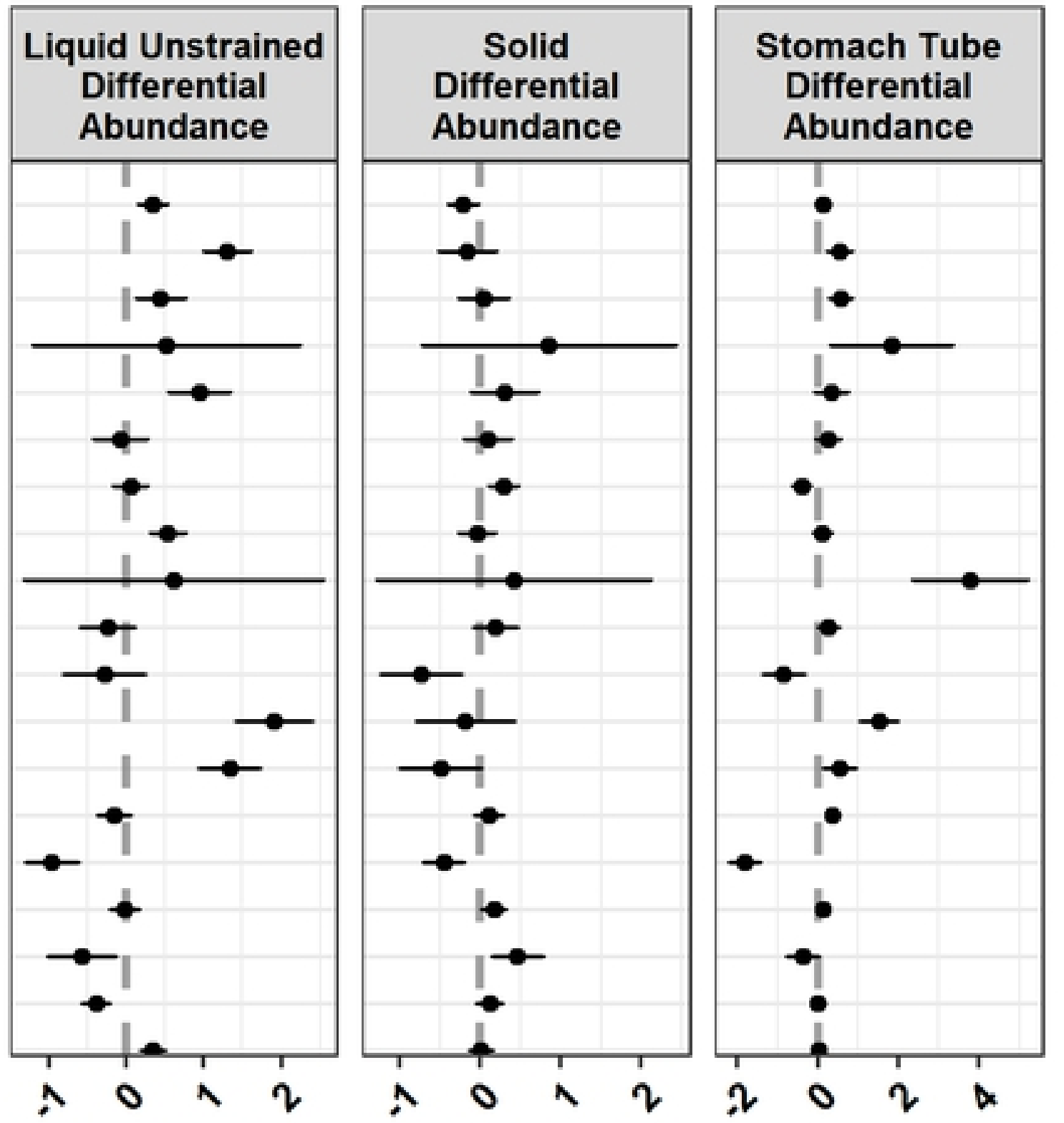

Fig 1 


\section{Weighted Unifrac}

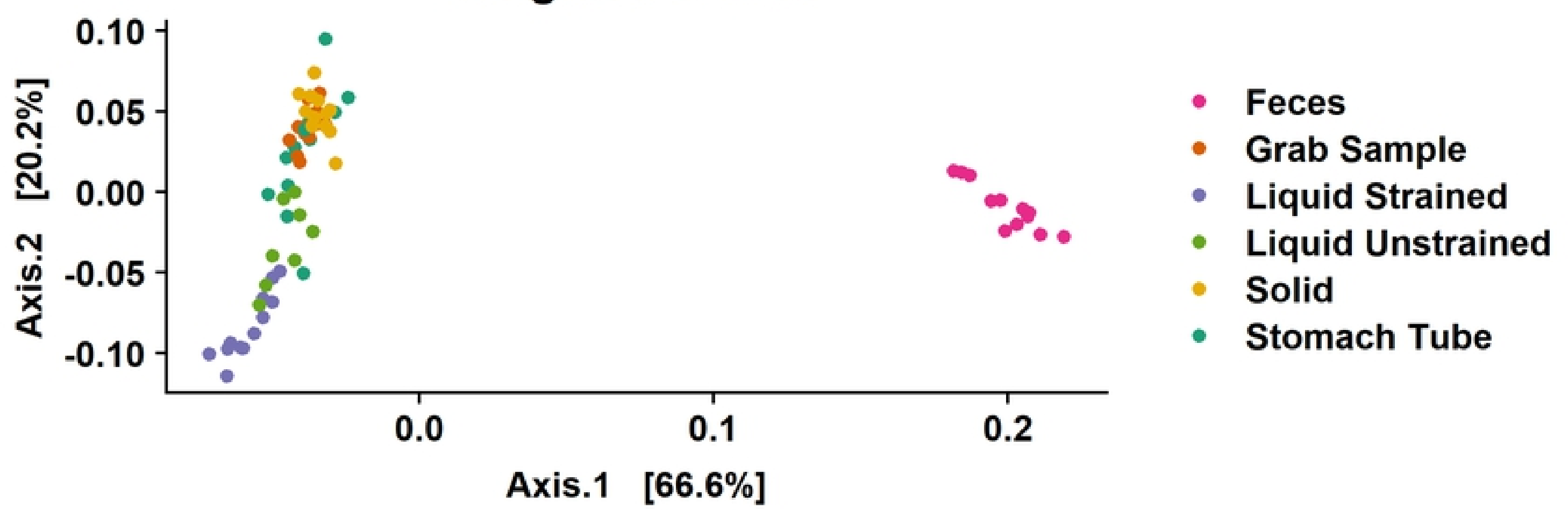

Fig 3 


\section{A Prevotellaceae}

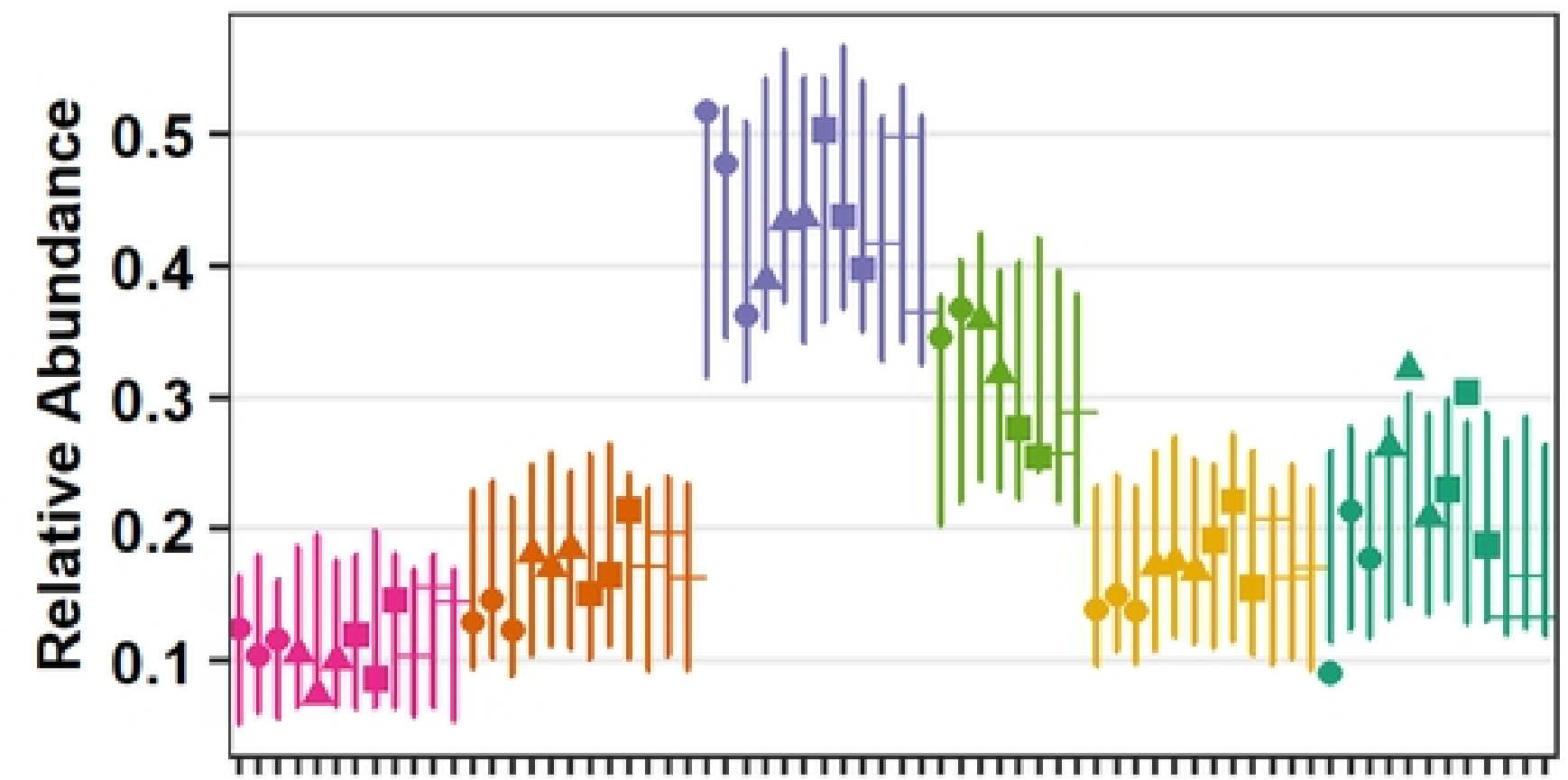

\section{B Ruminococcaceae}

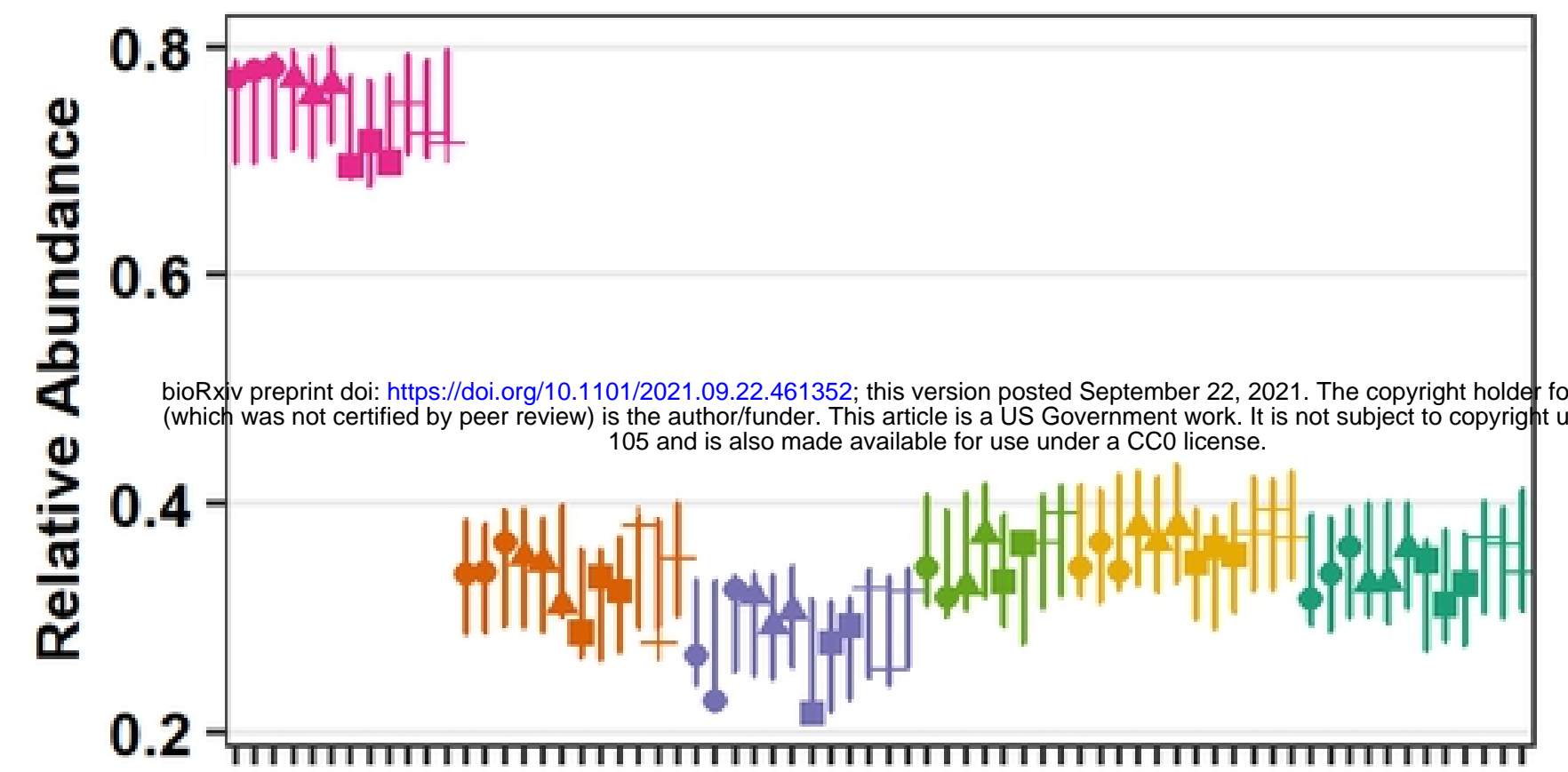

\section{Lachnospiraceae}

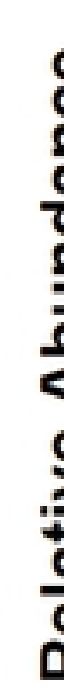

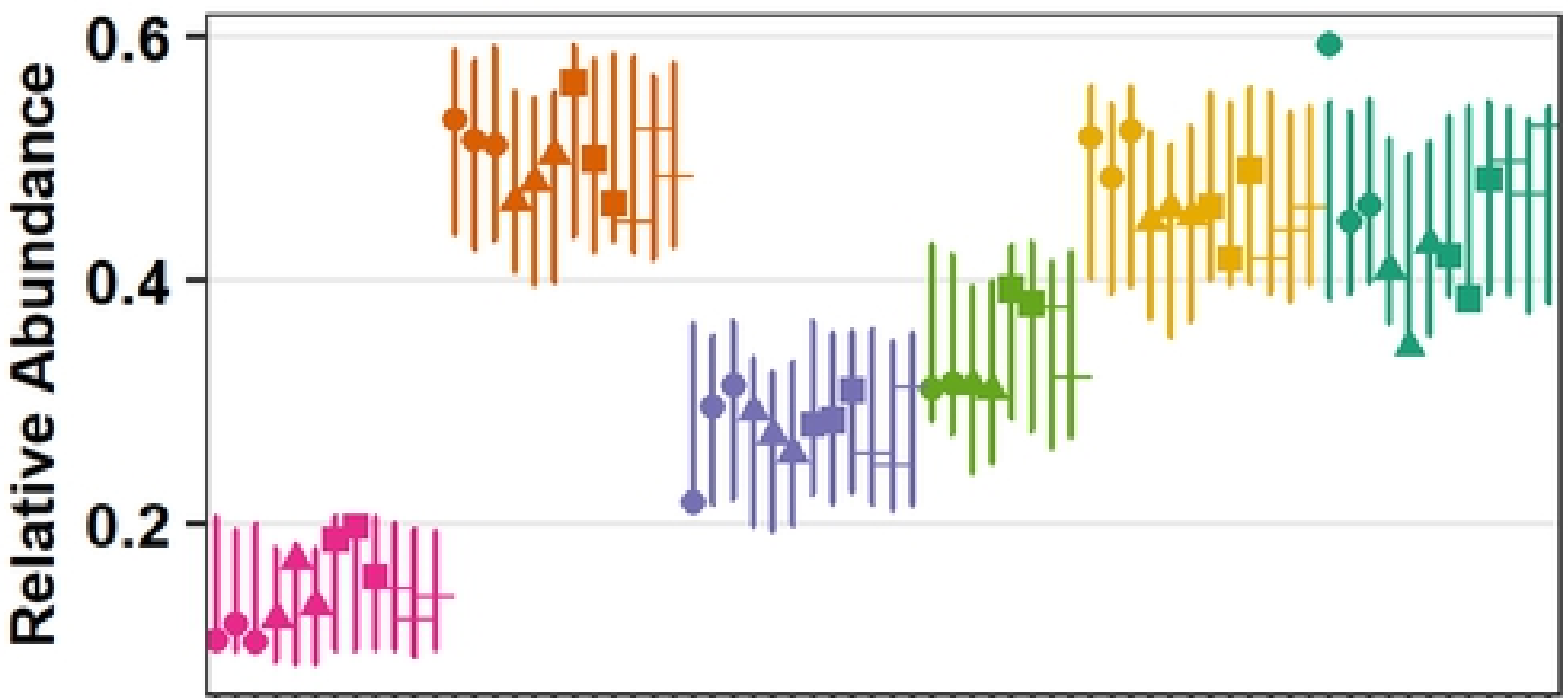

Samples

- Cow_2372

^ Cow_2477

- Cow_2549

+ Cow_796

$\rightarrow$ Grab Sample $\quad \rightarrow$ Liquid Strained

$\rightarrow$ Feces $\quad \rightarrow$ Liquid Unstrained

$\rightarrow$ Stomach Tube $\rightarrow$ Solid

Fig 5 


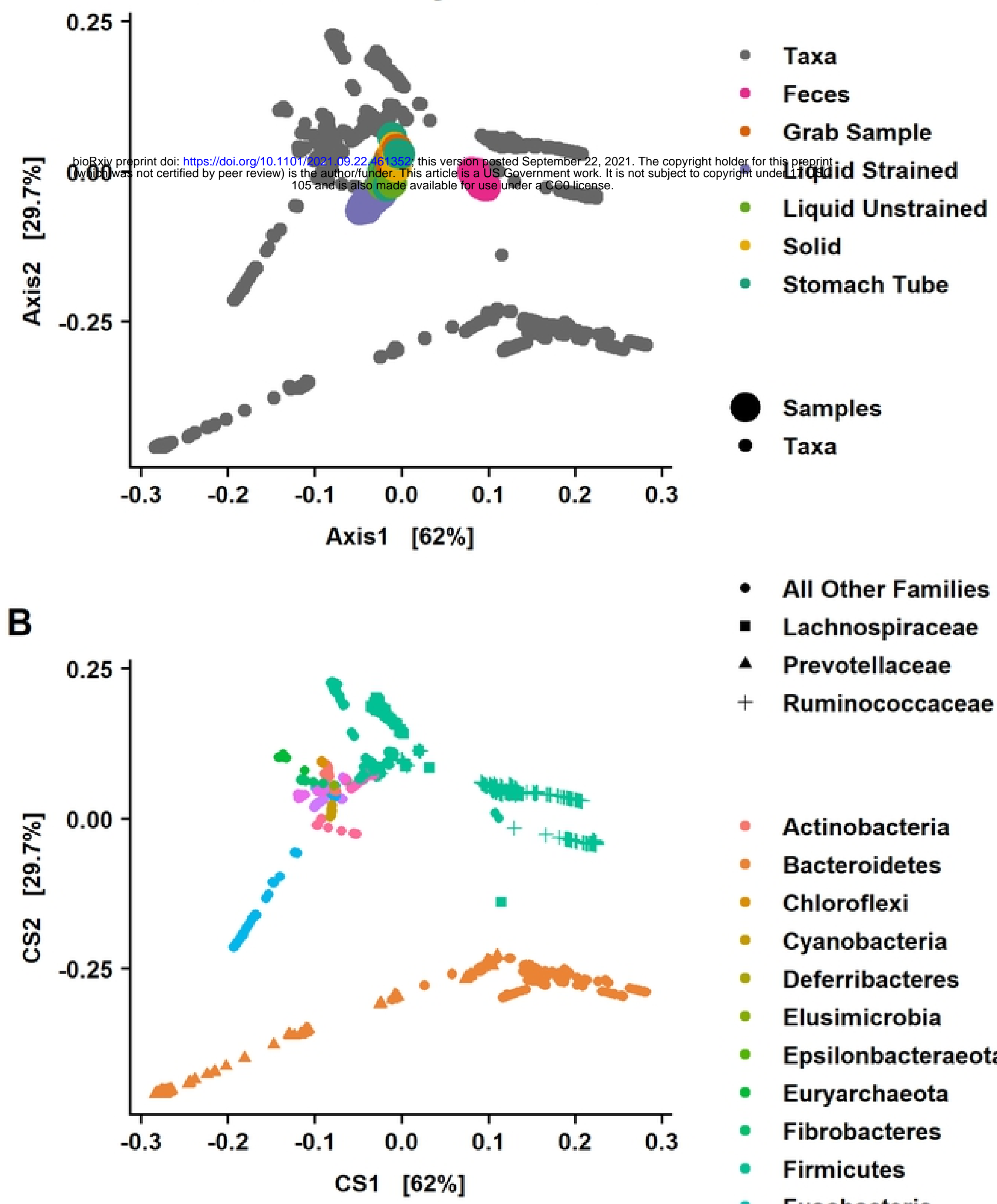

- Taxa

- Feces

- Grab Sample

- All Other Families

- Lachnospiraceae

- Prevotellaceae

+ Ruminococcaceae

- Actinobacteria
- Bacteroidetes
- Chloroflexi
- Cyanobacteria
- Eeferribacteres
- Elusimicrobia
- Epsilonbacteraeota
- Furyarchaeota
- Firmicutes
- Fusobacteria

- Gemmatimonadetes

- Kiritimatiellaeota

- Lentisphaerae

- Patescibacteria

- Planctomycetes

- Proteobacteria

- Spirochaetes

- Synergistetes

- Tenericutes

- Verrucomicrobia

Fig 4 
